Pacific Journal of Mathematics

ALGEBRAIC EXTENSIONS OF COMMUTATIVE BANACH 


\title{
ALGEBRAIC EXTENSIONS OF COMMUTATIVE BANACH ALGEBRAS
}

\author{
JOHN A. LINDBERG, JR.
}

1. Introduction. Let $A$ denote a commutative normed algebra with multiplicative unit and norm $\|\cdot\|$. In [2], Arens and Hoffman showed that it is possible to norm $A[x] /(\alpha(x))$, where $\alpha(x)=\sum_{i=0}^{n} \alpha_{i} x^{i}$ is a monic polynomial over $A$, in such a way that the canonical mapping of $A$ into $A[x] /(\alpha(x))$ is an isometry as well as an isomorphism; in fact, they give a family of norms on $A[x] /(\alpha())$, all of which are equivalent. Specifically, let $t$ be a positive number which satisfies $t^{n} \geqq$ $\left\|\alpha_{0}\right\|+\left\|\alpha_{1}\right\| t+\cdots+\left\|\alpha_{n-1}\right\| t^{n-1}$. Let $\sum_{i=0}^{n-1} a_{i} x^{i}+(\alpha(x))$ be any coset in $A[x] /(\alpha(x))$. As is well known, $\sum_{i=0}^{n-1} a_{i} x^{i}$ is the unique representative of this coset of lowest degree. Thus, $\left\|\sum_{i=0}^{n-1} a_{i} x^{i}+(\alpha(x))\right\|=\sum_{i=0}^{n-1}\left\|a_{i}\right\| t^{i}$ is well defined and makes $A[x] /(\alpha(x))$ into a normed algebra. Clearly, $a \rightarrow a+(\alpha(x)), a \in A$, is an isometry of $A$ into $A[x] /(\alpha(x)$ ). (Unless otherwise stated, we assume without loss of generality that $t=1$.) From the form of the norm we see that $A[x] /(\alpha(x))$ is a Banach algebra under this norm precisely when $A$ is a Banach algebra under $\|\cdot\|$. In the present paper, we deal mainly with the case where $A$ is a Banach algebra. In section nine we deal with, at some length, more general algebras.

In this paper we are mainly interested in the algebraic aspects of the extension $B=A[x] /(\alpha(x))$. However, we also present results which are Banach algebraic in nature. For example in section three we give a complete description of the Silov boundary of $B$. Section four is devoted to the study of the inheritance by $B$ of the Banach algebra properties of regularity and self-adjointness. In particular, we show that if $A$ is regular then $B$ is also regular. Self-adjointness is not always inherited as Example 4.3 shows. A sufficient condition (which is satisfied, for example, when the discriminant of $\alpha(x)$ is invertible) is given under which this property is inherited. (This condition states that the set $S(\alpha(x), A)$ of singular points of $\alpha(x)$ is empty. This means that the natural mapping of the carrier space of $B$ onto the carrier space of $A$ is a local homeomorphism with respect to the weak ${ }^{*}$ topologies. See section two for a complete discussion of this concept.)

In section five we once again make use of the condition that $\alpha(x)$ has no singular points. Theorem 5.2 states that if $A$ is semi-simple

Received January 17, 1962, and in revised form October 23, 1963.

This paper is based on portions of the author's Doctoral dissertation which was prepared under the guidance of Professor B. R. Gelbaum, University of Minnesota. The author was supported, in part, by the Office of Ordnance Research, under contract SAR G DA ORD 12, while at Yale University. 
and if $S(\alpha(x), A)=\phi$, then $B$ decomposes into the direct sum of a closed subalgebra of the form $A[b]$, with $\alpha(b)=0$, and the radical of $B$.

The next section is motivated by a well-known result in classical field theory. If $A$ is a field and $\alpha(x)$ an irreducible polynomial, then any root $b \in B$ of $\alpha(x)=0$ gives rise to an automorphism $\left(\sum_{i=0}^{n-1} a_{i} x^{i}+(\alpha(x)) \rightarrow\right.$ $\sum_{i=0}^{n-1} a_{i} b^{i}$ ) of $B$ which leaves invariant each element of $A$. In the present context this is no longer generally true. However, we are able to give two sets of conditions which assure us of this conclusion. Theorem 6.1 states that if $A[b]$ is dense in $B$, then $\sum_{i=0}^{n-1} a_{i} x^{i}+(\alpha(x)) \rightarrow$ $\sum_{i=0}^{n-1} a_{i} b^{i}$ is an automorphism. Theorem 6.2 requires that the discriminant $d$ of $\alpha(x)$ satisfy the condition that $d a \in \operatorname{Rad}(A)$ imply $a \in \operatorname{Rad}(A)(\operatorname{Rad}(A)$ denotes the radical of $A$ ) and that the Gelfand transform of $b$ satisfy a certain separation property. Also in section six we give conditions under which the automorphisms of $B$ which leave each element of $A$ invariant are periodic. The period is shown to be a factor of $n$ !, $n=$ degree of $\alpha(x)$ over $A$. Examples can be given which show that in the absence of any restrictions some of the automorphisms of $B$ leaving invariant each element of $A$ have infinite order.

In the next two sections we deal exclusively with polynomials over $A$ which have invertible discriminants in $A$. Section seven is concerned with the problem of extending a ring isomorphism of $A_{1}$ onto $A_{2}$ to an isomorphism of $A_{1}[x] /\left(\alpha_{1}(x)\right)$ onto $A_{2}[x] /\left(\alpha_{2}(x)\right)$. A necessary and sufficient condition is given under which such an extension exists. The extension is not necessarily unique. Prior to establishing this theorem we characterize those elements $b \in B$ such that $B=A[b]$ (= algebra of polynomials in $b$ with coefficients in $A$ ). Attention is given to extending involutions on $A$ to involutions of $B$.

In section eight we show that repeated extensions are again simple algebraic extensions (algebraically and topologically) of the type under discussion in this paper.

In the last section we give a complete description of the radical of $B$. The major results are stated for algebras over fields of characteristic zero. The main theorem (9.2) states that if $A$ is semi-simple, then the radical of $B$ is a nilpotent ideal. The degree of nilpotency is also specified. As a corollary, we have that if $B$ is semi-simple, then $A$ is semi-simple and the discriminant of $\alpha(x)$ is not a zero divisor in $A$, or zero. Applying this to the case of a tractable normed algebra (intersection of the closed maximal ideals is $(0)$ ), we show that the radical of $B$ and the intersection of the closed maximal ideals of $B$ coincide.

We now proceed to section two which contains some preliminaries gathered from other sources.

2. Preliminaries. If $A$ is a Banach algebra (always assumed to 
be commutative and to possess a multiplicative unit $e$ ), then $\Phi_{A}$ (called the carrier space of $A,[12]$ ) is to denote the space of (non-trivial) multiplicative linear functionals on $A$ to $C$ (= complex numbers). If $(h, \lambda) \in \Phi_{A} \times C$, then $(h, \lambda)$ can be considered as a multiplicative linear functional on $A[x]$, its action on elements $\Sigma a_{i} x^{i} \in A[x]$ being defined by $(h, \lambda) \Sigma a_{i} x^{i}=\Sigma h\left(a_{i}\right) \lambda^{i}$. In [2] it is shown that $\Phi_{B}, B=A[x] /(\alpha(x))$ (throughout this paper, $B$ will be used to denote $A[x] /(\alpha(x)), \alpha(x)$ monic), is (identifiable with) the set $\left\{(h, \lambda) \in \Phi_{A} \times C:(h, \lambda) \alpha(x)=0\right\}$. It should be noted that if $(h, \lambda) \in \Phi_{B}$, then $|\lambda| \leqq 1$ (recall that we are assuming $\sum_{i=0}^{n-1}\left\|\alpha_{i}\right\| \leqq 1$ so that $\left.\|x+(\alpha(x))\|=1\right)$. The coset $a+(\alpha(x))$ will be denoted by $a$ for $a \in A$ and $x+(\alpha(x))$ will be denoted by [x].

$x$ will be considered as an indeterminant over $\hat{A}$ (= Gelfand representation of $A$ ) and $C$ as well as an indeterminant over $A$. If $\beta(x)=$ $\Sigma \beta_{i} x^{i} \in A[x]$, then $\hat{\beta}(x)$ is to denote the polynomial $\Sigma \widehat{\beta}_{i} x^{i}$ over $\hat{A}$ and $\beta_{h}(x)$ is to denote the polynomial $\Sigma \widehat{\beta}_{i}(h) x^{i}$ over $C$. If $\beta(x) \in A[x]$ and $\beta_{h}(\lambda)=0, \lambda \in C$, but $\beta_{h}(x)$ not the zero polynomial, then we denote the multiplicity of $\lambda$ as a root of $\beta_{h}(x)=0$ by $M_{\beta}(h, \lambda)$. We call $M_{\beta}$ the multiplicity function of $\beta(x)$.

We include for the convenience of the reader several results that we will need from other sources.

2.1. $\pi$ defined by $\pi(h, \lambda)=h,(h, \lambda) \in \Phi_{B}$, is an open continuous mapping of $\Phi_{B}$ onto $\Phi_{A}$.

2.2. For each $h \in \Phi_{A}$ there are disjoint neighborhoods $V_{1}, \cdots, V_{m}$ in $\Phi_{B}$ of the points in $\pi^{-1}(h)=\left\{\left(h, \lambda_{1}\right), \cdots,\left(h, \lambda_{m}\right)\right\}$ such that $\pi\left(V_{1}\right)=$ $\pi\left(V_{i}\right), i=2, \cdots, m$, and $\pi^{-1}\left(\pi\left(V_{1}\right)\right)=\bigcup_{i=1}^{m} V_{i}$.

2.3. $M_{\alpha}$ is locally constant at $(h, \lambda) \in \Phi_{B}$ if and only if $\pi$ is a local homeomorphism at $(h, \lambda)$.

2.4. (Arens and Calderón) If $\beta(x) \in A[x]$ (not necessarily monic) and if $f \in C\left(\Phi_{A}\right)$ such that $\widehat{\beta}(f)=0$ but $\widehat{\beta}^{\prime}(f)$ never vanishes on $\Phi_{A}$ $\left(\beta^{\prime}(x)\right.$ is the formal derivative of $\left.\beta(x)\right)$, then a unique element $b \in A$ exists such that $\beta(b)=0$ and $\hat{b}=f$. (Arens and Calderón did not assert the uniqueness of $b$. However, it is easily established. Write $\beta(x)=$ $(x-b) Q(x), Q(x) \in A[x]$ and suppose $b^{\prime} \in A, \beta\left(b^{\prime}\right)=0$ and $\hat{b}^{\prime}=\hat{b}$. Then $\left(b^{\prime}-b\right) Q\left(b^{\prime}\right)=0$. Since $f(h)$ is a simple root of $\beta_{h}(x)=0, Q(b)^{\wedge}(h) \neq 0$ for every $h \in \Phi_{\Delta}$, so that $Q(b)$ is invertible in $A$. Hence $b=b^{\prime}$.)

Related to the above is

2.5. If $\alpha(x) \in A[x]$ is a monic polynomial, if $f \in C\left(\Phi_{A}\right)$ such that $\hat{\alpha}(f)=0$ and if $M_{\alpha}(\cdot, f(\cdot))$ is locally constant on $\Phi_{A}$, then $f \in \hat{A}$. (A stronger conclusion similar to the above can not be drawn here.) 
2.1, 2.2, 2.3 and 2.5 are proved in [10] while 2.4 is proved in [1].

Let $\alpha(x) \in A[x]$ be monic. If $h \in \Phi_{A}$ is such that each point of $\pi^{-1}(h)$ possesses a neighborhood on which $M_{\alpha}$ is constant, or what is equivalent (in view of 2.3), $\pi$ is a local homeomorphism at each point of $\pi^{-1}(h)$, then we call $h$ an ordinary point of $\alpha(x)$. If $h \in \Phi_{A}$ is not an ordinary point of $\alpha(x)$, we say that it is a singular point of $\alpha(x)$ and the set of such points will be denoted by $S(\alpha(x), A)$. It is clear that if $h \in \Phi_{A}$ is an ordinary point of $\alpha(x)$, then each $h^{\prime}$ sufficiently close to $h$ is also an ordinary point of $\alpha(x)$ so that $S(\alpha(x), A)$ must be a closed subset of $\Phi_{A} . \quad S(\alpha(x), A)$ is a subset of the set $D$ where $\hat{d}$ vanishes, where $d$ is the discriminant of $\alpha(x)$ (cf. [2] and page 93, [14]). (Note that $\hat{d}(h)$ is the discriminant of $\alpha_{h}(x)$.) $S(\alpha(x), A)$ can be null even if $D$ is not null. On the other hand, $S(\alpha(x), A)$ can be all of $D$. Because the cardinality of the sets $\pi^{-1}(h)$ is uniformly bounded by $n$ (=degree of $\alpha(x)), S(\alpha(x), A)$ is easily shown to be nowhere dense in $\Phi_{\Delta}$.

3. The Silov Boundary of $A[x] /(\alpha(x))$. Let $A^{\prime}$ be a Banach algebra extension of the Banach algebra $A$, let $\partial A, \partial A^{\prime}$ denote respectively the Silov boundaries of $A$ and $A^{\prime}$, and $\pi$ the natural mapping of $\Phi_{A^{\prime}}$ into $\Phi_{A}$ defined by $h=\pi\left(h^{\prime}\right)=h^{\prime} \mid A, h^{\prime} \in \Phi_{A}$. Then it is well known that $\pi\left(\partial A^{\prime}\right) \supset \partial A$. If $A^{\prime}$ is the extension $B=A[x] /(\alpha(x))$, then this result can be sharpened; indeed, we have that $\partial B=\pi^{-1}(\partial A)$. In the proof of this assertion, we need (Theorem 5, Appendix IV, [5]): A necessary and sufficient condition that $h_{0} \in \partial A$ is that for each neighborhood $V$ in $\Phi_{A}$ of $h_{0}$ there is a function $f \in \hat{A}$ whose absolute value $|f|$ attains its maximum (which we may assume is 1 ) on $V$ and is less than that on $\Phi_{A} \sim V$.

\section{THEOREM 3.1. $\partial B=\pi^{-1}(\partial A)$.}

Proof. We first show that $\pi^{-1}(\partial A) \subset \partial B$. Let $h_{0} \in \partial A$, let $W_{0}$ be a neighborhood in $\Phi_{B}$ of $\left(h_{0}, \lambda_{0}^{(1)}\right)$, and let $g \in B^{\wedge}$ such that $g\left(h_{0}, \lambda^{(1)}\right)=1$ and zero at the other points $\left(h_{0}, \lambda_{0}^{(i)}\right)$ of the fiber $\pi^{-1}\left(h_{0}\right)$. Let $W_{1} \subset W_{0}$ be an open neighborhood in $\Phi_{B}$ of $\left(h_{0}, \lambda_{0}^{(1)}\right)$ such that $|g(h, \lambda)|>1 / 2$ if $(h, \lambda) \in W_{1}$ and $W_{i}$ an open neighborhood in $\Phi_{B}$ of $\left(h_{0}, \lambda_{0}^{(i)}\right), i \neq 1$, such that $|g(h, \lambda)|<1 / 2$ if $(h, \lambda) \in W_{i}$. Since $\pi$ is an open mapping, $V_{0}=$ $\bigcap_{i} \pi\left(W_{i}\right)$ is an open neighborhood in $\Phi_{A}$ of $h_{0}$. Let $V_{i}=W_{i} \bigcap \pi^{-1}\left(V_{0}\right)$. Now, by the theorem quoted above, there is a function $f \in \hat{A}$ such that $\|f\|_{\infty}=1,\left|f\left(h_{1}\right)\right|=1, h_{1} \in V_{0}$, and $|f(h)|<1$ if $h \in \Phi_{A} \sim V_{0}$. Since $\Phi_{A} \sim V_{0}$ is closed, it is compact and hence there is a positive integer $N$ so large that

$$
|f(h)|^{N} \leqq \frac{1}{2\|g\|_{\infty}} \text { for } h \in \Phi_{A} \sim V_{0} .
$$


Then, if $h \notin V_{0}$ and $(h, \lambda) \in \Phi_{B}$, we have that

$$
\left|\left(f^{N} g\right)(h, \lambda)\right|=|f(h)|^{N}|g(h, \lambda)| \leqq \frac{1}{2\|g\|_{\infty}}\|g\|_{\infty}=\frac{1}{2},
$$

and if $(h, \lambda) \notin V_{1}$ but $h \in V_{0}$, we have that

$$
\left|\left(f^{N} g\right)(h, \lambda)\right|<|g(h, \lambda)| \leqq \frac{1}{2} .
$$

But for $\left(h_{1}, \lambda\right) \in V_{1}$,

$$
\left|\left(f^{N} g\right)\left(h_{1}, \lambda\right)\right|=\left|g\left(h_{1}, \lambda\right)\right|>\frac{1}{2} .
$$

Thus, $\left|f^{N} g\right|$ assumes its maximum value on $V_{1}$, and hence, on $W$, and is less than that outside $V_{1}$ or outside of $W$. By the above quoted theorem, $\left(h_{0}, \lambda_{0}^{(1)}\right) \in \partial B$, and $\pi^{-1}(\partial A) \subset \partial B$. We next show the reverse inclusion.

Let $\left(h_{0}, \lambda_{0}\right) \in \partial B$, and let $V$ be any neighborhood in $\Phi_{A}$ of $h_{0}$. Let $W$ be an open neighborhood in $\Phi_{B}$ of $\left(h_{0}, \lambda_{0}\right)$ such that $\pi(W) \subset V$ and no $\left(h_{0}, \lambda_{0}^{\prime}\right) \neq\left(h_{0}, \lambda_{0}\right)$ lies in $W$. Let $g \in \hat{B}$ be a function such that $\|g\|_{\infty}$ is assumed by $|g|$ on $W$ and $|g|<\|g\|_{\infty}$ outside of $W$. As in the above paragraph, we may assume that $|g(h, \lambda)|<1 / 2 n$ if $(h, \lambda) \in \Phi_{B} \sim$ $W$. Let $f$ be the function defined by

$$
f(h)=\sum_{i=1}^{n} g\left(h, \lambda_{i}(h)\right)
$$

where the $\lambda_{i}(h)$ denote all the roots (each distinct root repeated according to its multiplicity) of $\alpha_{h}(x)=0$. Then $f \in \hat{A}$. Now, for $h \notin \pi(W)$

$$
|f(h)|=\left|\sum_{i=1}^{n} g\left(h, \lambda_{i}\right)\right|<\sum_{i=1}^{n}\left|g\left(h, \lambda_{i}\right)\right|<\frac{1}{2} .
$$

There exists $\left(h_{1}, \lambda_{1}\right) \in W$ such that $\mid g\left(h_{1}, \lambda_{1}\right)_{1}=\|g\|_{\infty}$. (Assume that $\lambda_{1}=\lambda_{1}\left(h_{1}\right)$.) Then

$$
\begin{aligned}
\left|f\left(h_{1}\right)\right| & =\left|g\left(h_{1}, \lambda_{1}\right)+\sum_{i=2}^{n} g\left(h_{1}, \lambda_{i}(h)\right)\right| \\
& >\left|g\left(h_{1}, \lambda_{1}\right)\right|-\sum_{i=2}^{n}\left|g\left(h_{1}, \lambda_{i}(h)\right)\right|>1-\frac{n-1}{2 n}>\frac{1}{2} .
\end{aligned}
$$

Thus, $h_{0} \in \partial A$ and $\pi(\partial B) \subset \partial A$. Using the fact that $\pi^{-1}(\partial A) \subset \partial B$, we have that $\pi^{-1}(\partial A) \supset \partial B$. This completes the proof of the theorem.

4. Inheritance of the properties of regularity and self-adjointness. The properties of regularity and self-adjointness are possessed by many important and interesting Banach algebras and hence it is of interest 
to know whether these properties are inherited by the extension $B$. G. A. Heuer in [6] has shown that if $A$ is regular and self-adjoint and if in addition the discriminant of the monic polynomial $\alpha(x)$ is invertible in $A$, then $B$ is both regular and self-adjoint. In this section, we show that regularity is always inherited (without the assumption of self-adjointness). As a corollary we show that if $\hat{A}$ is dense in $C\left(\Phi_{A}\right)$, then $\hat{B}$ is dense in $C\left(\Phi_{B}\right)$. (For a discussion of the inheritance by $\hat{B}$ of the sup norm completeness of $\hat{A}$, the reader is referred to [7].) Example 4.3 shows that the self-adjointness of $A$ is not always inherited by $B$. We finally show that if $S(\alpha(x), A)=\phi$, then selfadjointness is inherited.

THEOREM 4.1. Let $A$ be a regular Banach algebra and let $\alpha(x)$ be a monic polynomial over $A$. Then $B$ is regular.

Proof. It suffices to show that if given $\left(h_{0}, \lambda_{0}\right) \in \Phi_{B}$ and a neighborhood $W$ in $\Phi_{B}$ of $\left(h_{0}, \lambda_{0}\right)$, then there exists a function $\hat{b} \in \hat{B}$ such that $\hat{b}\left(h_{0}, \lambda_{0}\right)=1$ and $\hat{b}(h, \lambda)=0$ if $(h, \lambda) \in \Phi_{B} \sim W$. From 2.2, it follows that there is a neighborhood $V$ in $\Phi_{A}$ of $h_{0}$ so small that $V \subset \pi(W)$ and $\pi^{-1}(V)=\bigcup_{i=1}^{m} V_{i}$ where the $V_{i}$ are disjoint neighborhoods of the points in $\pi^{-1}\left(h_{0}\right)$ with $W \supset V_{1}$. We assume (without loss of generality) that the sets $V_{1}, \cdots, V_{m}$ are closed. Since $A$ is regular the set $V$ is hull-kernel closed in $\Phi_{A}$, from which it follows that $\pi^{-1}(V)$ is hull-kernel closed in $\Phi_{B}$. Now, let $I$ denote the ideal in $B$ of elements whose transforms in $\hat{B}$ vanish on $\pi^{-1}(V)$. Since $I$ is a closed ideal, $B / I$ is a Banach algebra with carrier space (identifiable with) $\pi^{-1}(V)$ (cf. [11]). By [13], there is an idempotent $f$ in $B / I$ such that $\hat{f}(h, \lambda)=1$ if and only if $(h, \lambda) \in V_{1}$. But $\hat{f}=\hat{f}_{0} \mid \pi^{-1}(V)$ for some $f_{0} \in B$. Since $A$ is regular there is an element $a \in A$ such that $\hat{a}\left(h_{0}\right)=1$ and $\hat{a}$ vanishes outside of $V$. Then $b=a f_{0}$ is an element of $B$ such that $\hat{b}\left(h_{0}, \lambda_{0}\right)=1$ and $\hat{b}(h, \lambda)=0$ outside of $V_{1} \subset W$. This completes the proof of the theorem.

The corollary below extends the following result of Heuer [6]: If $\hat{A}$ is dense in $C\left(\Phi_{A}\right)$ and if for each singular point $h, \pi^{-1}(h)$ consists of exactly one point, then $\hat{B}$ is dense in $C\left(\Phi_{B}\right)$. The proof given below is essentially due to Heuer.

Corollary 4.2. If $A$ is a Banach algebra and if $\hat{A}$ is dense in $C\left(\Phi_{A}\right)$, then $\hat{B}$ is dense in $C\left(\Phi_{B}\right)$.

Proof. Since $\hat{A}$ is dense in $C\left(\Phi_{A}\right)$, it is easily shown that $\hat{B}=$ $(\hat{A}[x] /(\hat{\alpha}(x)))^{\wedge}$ is dense in $B_{0}=\left(C\left(\Phi_{A}\right)[x] /(\widehat{\alpha}(x))\right)^{\wedge}$, with both algebras being viewed as subalgebras of $C\left(\Phi_{B}\right)$. Thus, it suffices to show that $B_{0}$ is dense in $C\left(\Phi_{B}\right)$. (It need not be the case that $B_{0}$ is all of $C\left(\Phi_{B}\right)$ 
as Example 4.3 of this section shows.) Let $h \in \Phi_{A}$ be arbitrarily given. By the theorem, $B_{0}$ is regular so that if $V_{1}, \cdots, V_{m}$ are disjoint neighborhoods of the points in $\pi^{-1}(h)$, then there exists a function $f \in B_{0}$ which takes the value $i$ on $V_{i}, i=1,2, \cdots, m$. Let $g$ be a real-valued function in $C\left(\Phi_{A}\right)$ such that $g(h)=1$ and $g$ vanishes outside of $\bigcap_{i=1}^{m} \pi\left(V_{i}\right)$. Then $(g f)^{\wedge}$ is a real-valued function in $B_{0}$ which separates the points of $\pi^{-1}(h)$. Since $C\left(\Phi_{A}\right)$ is (isomorphic to) a subalgebra of $B_{0}$, any two points $(h, \lambda),\left(h^{\prime}, \lambda^{\prime}\right) \in \Phi_{B}$, with $h \neq h^{\prime}$, can be separated by a real-valued function in $B_{0}$. Hence any two points in $\Phi_{B}$ can be separated by a real-valued function in $B_{0}$. The conclusion of the corollary now follows from the Stone-Weierstrass Theorem.

We now turn our attention to the question of inheritance of the property of self-adjointness, and first give an example which shows that this property is not always inherited by the extension.

ExAmple 4.3. Let $A=C(\Delta), \Delta=\{z \in C:|z| \leqq 1\}$ and $\alpha(x)=x^{2}-f_{0}$, $f_{0}(z) \equiv z$. Then $A[x] /(\alpha(x))$ is not self-adjoint. For if it were, then $\left([x]^{\wedge}\right)^{-}=\hat{a}_{0}+\hat{a}_{1}[x]^{\wedge}$ for some choice of $a_{0}, a_{1} \in A$. But this means that $\widehat{a}_{1}(z)=\exp (-\arg z), z \neq 0$. This is a contradiction since $\exp (-\arg z)$ is not extendable to a continuous function on $\Delta$.

THEOREM 4.4. Let $A$ be a self-adjoint Banach algebra and $\alpha(x)=$ $\sum_{i=0}^{n} \alpha_{i} x^{i}$ be a monic polynomial over $A$. If $S(\alpha(x), A)=\phi$, then $A[x] /(\alpha(x))$ is self-adjoint.

Proof. Let $f(h, \lambda) \equiv \bar{\lambda}$ for $(h, \lambda) \in \Phi_{B}$. Then $f \in C\left(\Phi_{B}\right)$ and $\hat{\beta}(f)=$ 0 , where $\beta(x)=\sum_{i=0}^{n} \beta_{i} x^{i}, \widehat{\beta}_{i}=\left(\widehat{\alpha}_{i}\right)^{-}, i=0,1, \cdots, n-1$, and $\beta_{n}=e$. Since the multiplicity function $M_{\alpha}$ of $\alpha(x)$ is locally constant on $\Phi_{B}$, it follows that $M_{\beta}(\cdot, f(\cdot))$ is locally constant on $\Phi_{B}$, where $M_{\beta}$ is the multiplicity function of $\beta(x)$ when viewed as a polynomial over $B$. By 2.5 , it follows that $f \in \hat{B}$ so that $B$ is self-adjoint since $\left(\sum \widehat{a}_{i}\left([x]^{\wedge}\right)^{i}\right)^{-}=$ $\sum\left(\hat{a}_{i}\right)^{-} f^{i} \in \hat{B}$.

5. On the Wedderburn decomposition of $B$. In this section we discuss the Wedderburn decomposition of the extension $B$, that is, the decomposition of $B$ into the direct sum of a closed subalgebra $B_{0}$ of $B$ and the radical $\operatorname{Rad}(B)$ of $B\left(B=B_{0} \oplus \operatorname{Rad}(B)\right)$. As is well known, such a decomposition in general does not hold for Banach algebras, even in the weaker sense where one does not require that the subalgebra be closed. We will give an example which supports this statement. Badé and Curtis have given an example in [3]. Feldman, in [4], gave an example where the stronger Wedderburn decomposition failed to hold. For this example, the weaker decomposition holds. 
The condition that $S(\alpha(x), A)=\phi$ (A semi-simple) is sufficient for such a decomposition of $B$ to hold. When this condition holds, $\alpha(x)$ is forced to factor; precisely, there exist mutually orthogonal idempotents $e_{1}, \cdots, e_{m}$, positive integers $k_{i j}$, and polynomials $\alpha_{i j}(x) \in A[x], j=$ $1, \cdots, S_{i} ; i=1, \cdots, m$, such that $e_{i} \alpha_{i j}(x)$ is monic over $e_{i} A$, the discriminant of $\prod_{j=1}^{S_{i}} e_{i} \alpha_{i j}(x)$ is invertible in $e_{i} A$, and

$$
\alpha(x)=\sum_{i=1}^{m} e_{i} \prod_{j=1}^{s_{i}} \alpha_{i j}(x)^{k_{i j}} .
$$

Furthermore, the radical of $A[x] /(\alpha(x))$ is a principal ideal generated by $\beta([x])$, where $\beta(x)=\sum_{i=1}^{m} e_{i} \prod_{j=1}^{s_{i}} \alpha_{i j}(x)$ (cf. Theorem 2.3, [10]).

THEOREM 5.2. Let $A$ be a semi-simple Banach algebra and $\alpha(x)$ a monic polynomial over $A$. If $S(\alpha(x), A)=\phi$, then there exists an element $b \in B$ such that $\alpha(b)=0, A[b]$ is closed in $B$ and $B=A[b] \oplus$ $\operatorname{Rad}(B)$.

Proof. To simplify the proof, we first assume that $m=1$ in the above paragraph. Thus, $\alpha(x)$ is of the form $\prod_{i=1}^{S} \alpha_{i}(x)^{k_{i}}$, where each $\alpha_{i}(x)$ is monic over $A$ and $\beta(x)=\prod_{i=1}^{S} \alpha_{i}(x)$ has an invertible discriminant in $A$.

Since $\beta\left([x]^{\wedge}\right)=0$ and since $\beta(x)$ has an invertible discriminant in $A$, and hence in $B$, there exists an element $b \in B$ such that $\beta(b)=0$ and $\hat{b}=[x]^{\wedge}$. Thus, $\alpha(b)=0$ also. Since $\hat{b}=[x]^{\wedge}$, there is an element $R \in \operatorname{Rad}(B)$ such that $[x]=b+R$ so that $\sum_{i=0}^{n-1} a_{i}[x]^{i}=\sum_{i=0}^{n-1} a_{i} b^{i}+$ (polynomial in $R$, with zero constant term) ( $n=$ degree of $\alpha(x)$ ). Hence, $B$ is the sum of $A[b]$ and $\operatorname{Rad}(B)$. We next show that the sum is a direct sum. Let $t$ be the degree of $\beta(x)$ over $A$. Then $\sum_{i=0}^{n-1} a_{i}^{\prime} b^{i}$ can be expressed in the form $\sum_{i=0}^{t-1} a_{i} b^{i}$ for some choice of $a_{0}, \cdots, a_{t-1}$ in $A$. Suppose now that $\sum_{i=0}^{t-1} a_{i} b^{i} \in \operatorname{Rad}(B)$. Then $\sum_{i=0}^{t-1} a_{i} x^{i}$ is a multiple of $\beta(x)$ (this follows since the radical of $B$ is a principal ideal generated by $\beta([x]))$. Thus, the $a_{i}$ 's must all be 0 . Thus, the sum is direct. (Note also that $\sum_{i=0}^{t-1} a_{i} b^{i}=0$ if and only if $a_{i}=0, i=0,1, \cdots, t-1$.)

In order to show that $A[b]$ is closed, we introduce a mapping $\phi$ of $B$ onto $A[y] /(\beta(y))$ as follows: $\phi\left(\Sigma a_{i}[x]^{i}\right)=\Sigma a_{i}[y]^{i} . \quad \phi$ is well defined and a homomorphism since $\alpha([y])=0$. Furthermore, $\phi$ is continuous since

$$
\begin{aligned}
& \left\|\phi\left(\sum_{i=0}^{n-1} a_{i}[x]^{i}\right)\right\|=\left\|\mid \sum_{i=0}^{n-1} a_{i}[y]^{i}\right\| \leqq \sum_{i=0}^{n-1}\left\|a_{i}\right\|\|[y]\|^{i} \\
& \leqq K \sum_{i=0}^{n-1}\left\|\alpha_{i}\right\|=K\left\|\sum_{i=0}^{n-1} a_{i}[x]^{i}\right\|
\end{aligned}
$$

where $K=\max \left\{1,\|[y]\|, \cdots,\|[y]\|^{n-1}\right\}$. Since $\operatorname{Rad}(B)$ is generated by $\beta([x]), \phi(\operatorname{Rad}(B))=0$. But $[x]-b \in \operatorname{Rad}(B)$ so that $\phi(b)=\phi([x])=[y]$. 
Thus, if $\sum_{i=0}^{t-1}\left\|\beta_{i}\right\| k^{i} \leqq k^{t}$, where $\beta(y)=\sum_{i=0}^{t} \beta_{i} x^{i}$, then

$$
\sum_{i=0}^{t-1}\left\|a_{i}\right\| k^{i}=|| \sum_{i=0}^{t-1} a_{i}[y]^{i}|| \leqq K|| \sum_{i=0}^{t-1} a_{i} b^{i}\left\|\leqq K^{\prime} \sum_{i=0}^{t-1}\right\| a_{i} \|
$$

where $K^{\prime}=K \max \left\{1,\|b\|, \cdots,\|b\|^{t-1}\right\}$. Since $A$ is complete, the norm on $B$ restricted to $A[b]$ is complete or equivalently, $A[b]$ is closed in $B$. This completes the proof of the theorem if we assume that $m=1$.

The general situation follows immediately from what was proved above and the following observations. Let $e_{1}, e_{2}, \cdots, e_{m}$ be the idempotents which appear in the factorization of $\alpha(x)$ which was displayed in 5.1. Then $A=e_{1} A \oplus \cdots \oplus e_{m} A$ and $B=e_{1} B \oplus \cdots \oplus e_{m} B$, the direct sums being topological. Since the natural isomorphism $\phi_{i}$ of $e_{i} B$ onto $B_{i}=\left(e_{i} A\right)[x] /\left(e_{i} \alpha(x)\right)$ is $b i$-continuous and since $\operatorname{Rad}\left(B_{i}\right)=\phi_{i}\left(e_{i} \operatorname{Rad}(B)\right)$, it follows from the above that there exists $b_{i} \in e_{i} B$ such that $e_{i} \alpha\left(b_{i}\right)=$ $0,\left(e_{i} A\right)\left[b_{i}\right]$ is closed in $e_{i} B$ and $e_{i} B=\left(e_{i} A\right)\left[b_{i}\right] \oplus e_{i}(\operatorname{Rad} B)$. If we set $b=\sum_{i=1}^{m} b_{i}$, then $\alpha(b)=0, A[b]$ is closed in $B$ and $B=A[b] \oplus \operatorname{Rad}(B)$. This completes the proof of the theorem.

We now present an example that shows if we drop the condition that $S(\alpha(x), A)=\phi$, then the conclusion of Theorem 5.2 is not assured.

ExAMPLE 5.3. Take $A$ to be the algebra of functions $f$ which are continuous on the disc $\Delta=\{z \in C:|z| \leqq 1\}$, analytic in the interior of $\Delta$ and $f^{\prime}(0)=0$. For $\alpha(x)$, take $\left(x-f_{0}\right)^{2}\left(x+2 f_{0}\right)$ where $f_{0}(z) \equiv z$, $z \in \Delta$. Then $\alpha(x) \in A[x]$ and $S(\alpha(x), A)=\{0\} . \quad\left(\Phi_{A}\right.$ is identifiable with 4.) Now, there is no subalgebra $B_{0}$ of $B$ isomorphic to $B^{\wedge}$. (If $B=$ $B_{0} \oplus \operatorname{Rad}(B)$, then $B_{0} \cong \hat{B} \cong B / \operatorname{Rad}(B)$.) For if this were the case, then $B_{0}$ would coincide with $A[b]$ for some $b \in B$ and $b$ would have to satisfy $f_{0}^{2}\left(b-f_{0}\right)\left(b+2 f_{0}\right)=0$. This is easily shown to be impossible. It follows from Theorem 9.2 that the degree of nilpotency of $\operatorname{Rad}(B)$ is two.

6. Automorphisms and conjugate roots. If $g: A[x] /(\alpha(x)) \rightarrow$ $A[x] /(\alpha(x))$ is an automorphism such that $g(a)=a$ for all $a \in A$, then $g([x])$ is obviously a root of $\alpha(x)=0$ and $A[g([x])]=A[x] /(\alpha(x))$. Conversely, if $\alpha(b)=0, b \in A[x] /(\alpha(x))$, need the homomorphism $g: \Sigma a_{i}[x]^{i} \rightarrow$ $\Sigma a_{i} b^{i}$ be an automorphism of $B$ ? The answer is no in general (recall Theorem 5.2). However, there are various conditions (see 6.1 and 6.3) under which such homomorphisms $g$ are automorphisms. In 6.4 we give conditions under which automorphisms of the above type are periodic. We begin with

THEOREM 6.1. Let $\alpha(x)$ be a monic polynomial over the Banach algebra $A$. If $b \in B$ such that $A[b]$ is dense in $B$ and $\alpha(b)=0$, then $g: \sum_{i=0}^{n-1} a_{i}[x]^{i} \rightarrow \sum_{i=0}^{n-1} a_{i} b^{i}$ is an automorphism. 
Proof. What we actually prove is this: if $T$ is a linear transformation of $A^{n}=A \times \cdots \times A$ onto a dense subset of $A^{n}$ such that $a \cdot T\left(a_{1}, \cdots, a_{n}\right)=T\left(a a_{1}, \cdots, a a_{n}\right)$, then $T$ is one-to-one and onto. For a norm in $A^{n}$ we take $\left\|\left(a_{1}, \cdots, a_{n}\right)\right\|=\sum_{i=1}^{n}\left\|a_{i}\right\|$. (Clearly the homomorphism $g$ has these properties; note that as a Banach space $B=A^{n}$.)

Let $h \in \Phi_{A}$ and let $T_{h}$ denote the mapping $T_{h}:\left(h\left(a_{1}\right), \cdots, h\left(a_{n}\right)\right) \rightarrow$ $\left(h\left(a_{1}^{\prime}\right), \cdots, h\left(a_{n}^{\prime}\right)\right)$ where $\left(a_{1}^{\prime}, \cdots, a_{n}^{\prime}\right)=T\left(a_{1}, \cdots, a_{n}\right)$. Clearly, $T_{h}$ is a linear transformation of $C^{n}$ into itself since $C \cong A / h^{-1}(0)$. (For a norm in $C^{n}$, we take $\left.\left|\left(\lambda_{1}, \cdots, \lambda_{n}\right)\right|=\sum_{i=1}^{n}\left|\lambda_{i}\right|.\right) \quad$ Now, $T_{h}\left(C^{n}\right)$ must be dense in $C^{n}$. For if $\left(\lambda_{1}, \cdots, \lambda_{n}\right),\left(\mu_{1}, \cdots, \mu_{n}\right) \in C^{n}$, then there are elements $a_{i}, b_{i} \in A$ such that $h\left(a_{i}\right)=\lambda_{i}$ and $h\left(b_{i}\right)=\mu_{i}, i=1, \cdots, n$. If $\left(a_{1}^{\prime}, \cdots, a_{n}^{\prime}\right)=$ $T\left(a_{i}, \cdots, a_{n}\right)$, then

$$
\begin{aligned}
& \left|T_{h}\left(\lambda_{1}, \cdots, \lambda_{n}\right)-\left(\mu_{1}, \cdots, \mu_{n}\right)\right| \\
& \quad=\left|\left(h\left(a_{1}^{\prime}\right), \cdots, h\left(a_{n}^{\prime}\right)\right)-\left(h\left(b_{1}\right), \cdots, h\left(b_{n}\right)\right)\right| \\
& \quad=\sum_{i=1}^{n}\left|h\left(a_{i}^{\prime}\right)-h\left(b_{i}\right)\right| \leqq \sum_{i=1}^{n}\left\|a_{i}^{\prime}-b_{i}\right\| \\
& \quad=\left\|T\left(a_{1}, \cdots, a_{n}\right)-\left(b_{1}, \cdots, b_{n}\right)\right\| .
\end{aligned}
$$

It follows from the above that $T_{h}\left(C^{n}\right)$ is dense in $C^{n}$. But this means that $T_{h}$ is one-to-one and hence onto.

Now, consider $n$-linear equations in $a_{i}$ (considered to be unknowns) represented by

$$
\sum_{i=1}^{n} a_{i} T\left(e_{i}\right)=\left(b_{1}, \cdots, b_{n}\right),
$$

where $e_{i}$ is the vector in $A^{n}$ with $\boldsymbol{e}$ in the $i$ th place and zero elsewhere. If $D$ is the determinant of the matrix of the coefficients of system $(*)$, then $h(D)$ is precisely the determinant of the matrix associated with the linear transformation $T_{h}$. Since $T_{h}$ is onto, $h(D) \neq 0$.

Since $h \in \Phi_{A}$ in the above argument is quite arbitrary, $h(D) \neq 0$ for all $h \in \Phi_{A}$ so that $D$ is invertible in $A$. But this means that (*) has a unique solution $\left(a_{1}, a_{2}, \cdots, a_{n}\right) \in A$ for each $\left(b_{1}, \cdots, b_{n}\right) \in A^{n}$. Hence $T$ is both one-to-one and onto.

Let $G(B: A)$ denote the group of automorphisms of $B$ which leave invariant each element of $A$. If $g \in G(B: A)$, let $g^{*}$ denote the homeomorphism of $\Phi_{B}$ onto itself which satisfies $g(b)^{\wedge}(h, \lambda)=\hat{b}\left(g^{*}(h, \lambda)\right)$ for all $b \in B$ and all $(h, \lambda) \in \Phi_{B}$ (cf. [11]). $E\left(\Phi_{B}: \Phi_{A}\right)$ is to denote the group of homeomorphisms $\phi$ of $\Phi_{B}$ onto itself such that $\pi \circ \phi=\pi$.

Lemma 6.2. If $g \in G(B: A)$, then $g^{*}(h, \lambda)=\left(h, g([x])^{\wedge}(h, \lambda)\right)$ for every $(h, \lambda) \in \Phi_{B}$ and consequently $g^{*} \in E\left(\Phi_{B}: \Phi_{A}\right)$. Also, $\left(g^{*}\right)^{n \prime}=$ identity homeomorphism $(n=$ degree of $\alpha(x))$.

Proof. By the definition of $g^{*}$, we know that for $a \in A$ and 
$(h, \lambda) \in \Phi_{B}, \hat{a}(h)=\hat{a}(h, \lambda)=g(\alpha)^{\wedge}(h, \lambda)=\hat{a}\left(g^{*}(h, \lambda)\right)=\hat{a}\left(h^{\prime}\right)$, where $\left(h^{\prime}, \lambda^{\prime}\right)=$ $g^{*}(h, \lambda)$. Since $\hat{A}$ is a separating algebra of functions on $\Phi_{\Delta}$, it follows that $h=h^{\prime}$. Thus, $g^{*}(h, \lambda)=\left(h, \lambda^{\prime}\right)$ or equivalently, $\pi \circ g^{*}=\pi$. The last assertion of the lemma follows from the fact that if $\phi \in E\left(\Phi_{B}: \Phi_{A}\right)$, then $\phi \mid \pi^{-1}(h)$ is a permutation of $\pi^{-1}(h)$ so that $\phi^{n !}$ must be the identity homeomorphism on $\Phi_{B}$.

THEOREM 6.3. Let $\alpha(x)$ be a monic polynomial over the Banach algebra $A$. If the discriminant $d$ of $\alpha(x)$ has the property that $d a \in \operatorname{Rad}(A)$ implies that $a \in \operatorname{Rad}(A)$ and if $b \in B, \alpha(b)=0$ and $\hat{b}$ separates the points of $\pi^{-1}(h)$ for each $h \in \Phi_{A}$, then $g: \sum_{i=0}^{n-1} a_{i}[x]^{i} \rightarrow$ $\sum_{i=0}^{n-1} a_{i} b^{i}$ is an automorphism.

Proof. Corresponding to the homomorphism $g: \sum_{i=0}^{n-1} a_{i}[x]^{i} \rightarrow \sum_{i=0}^{n-1} a_{i} b^{i}$, let $\phi$ denote the mapping $\phi(h, \lambda)=\left(h, g([x])^{\wedge}(h, \lambda)\right)$. Since $\hat{b}=g([x])^{\wedge}$ separates the points of each fiber $\pi^{-1}(h), \phi$ is one-to-one and onto. Hence, $\phi \in E\left(\Phi_{B}: \Phi_{A}\right)$. For each $i$, it is easily shown that $\phi^{i}(h, \lambda)=$ $\left(h,\left(g^{i}([x])\right)^{\wedge}(h, \lambda)\right)$ for each $(h, \lambda) \in \Phi_{B}$. Thus, we have that $\phi^{n !}$ is the identity homeomorphism on $\Phi_{B}$. It now follows that $g^{n !}([x])^{\wedge}=[x]^{\wedge}$ or equivalently, $g^{n !}([x])-[x] \in \operatorname{Rad}(B)$.

Let $T=g^{n !}$. Then $T([x])-[x] \in \operatorname{Rad}(B)$. It further follows that for each $i=0, \cdots, n-1, T\left([x]^{i}\right)-[x]^{i} \in \operatorname{Rad}(B)$. Since $d a \in \operatorname{Rad}(A)$ implies that $a \in \operatorname{Rad}(A)$, where $d$ is the discriminant of $a(x), \hat{d}$ is not a zero divisor in $\hat{A}$ and $\operatorname{Rad}(B)=(\operatorname{Rad}(A))[[x]]$ (cf. [2]). Thus, there exist elements $r_{i j} \in \operatorname{Rad}(A), i, j=0, \cdots, n-1$, such that $T\left([x]^{i}\right)=$ $[x]^{i}+\sum_{j=0}^{n-1} r_{i j}[x]^{j}$. When $T$ is viewed as a linear transformation on $A^{n}$, the determinant associated with $T$ is invertible in $A$ so that $T$ is one-to-one and onto. But then $g$ must also be one-to-one and onto. This completes the proof of the theorem.

CoRollary 6.4. Maintain the hypothesis (on d) of the theorem. If either

(i) $\operatorname{Rad}(A)$ is a nilpotent ideal and $d$ is not a zero divisor in $A$, or

(ii) there exists $\mu>0$ such that $\|d r\| \geqq \mu\|r\|$ for all $r \in \operatorname{Rad}(A)$, obtains, then each $g \in G(B: A)$ is periodic; in fact, if $\left(g^{*}\right)^{p}$ is the identity homeomorphism, then $g^{p}$ is the identity automorphism of $B$.

Proof. From the theorem we know that $g^{p}([x])-[x]=R \in \operatorname{Rad}(B)$ if $\left(g^{*}\right)^{p}$ is the identity homeomorphism. We will show that if either (i) or (ii) obtains, then $R=0$ so that $g^{p}([x])=[x]$. If case (i) obtains, then $\operatorname{Rad}(B)$ is a nilpotent ideal (by Corollary 9.4). If we write $\alpha(y)=$ $(y-[x]) Q(y), Q(y) \in B[y]$, then $R \cdot Q([x]+R)=0$. Now there are elements $b_{i} \in B, i=1, \cdots, n-1$, such that $Q([x]+R)=\alpha^{\prime}([x])+\sum_{i=1}^{n-1} b_{i} R^{i}$ 
(by direct computation). If $R^{m} \neq 0$ but $R^{m+1}=0$, then $R^{m} \alpha^{\prime}([x])=0$. If we write $\alpha(y) s(y)+\alpha^{\prime}(y) t(y)=d, s(y), t(y) \in A[y]$ (cf. formula 4, page $96,[14])$, then $\alpha^{\prime}([x]) t([x])=d$. Thus, $R^{m} \cdot d=0$; hence $R^{m}=0$. This is a contradiction so that $R=0$.

Suppose next that case (ii) obtains. We first show that $d$ is not a zero divisor in $A$. For if $d a=0$, then we know that $a \in \operatorname{Rad}(A)$. But $0=\|d a\| \geqq \mu\|a\|$ and hence $a=0$. Now, as in the above, we have that $R \cdot Q([x]+R)=0$ or $R \cdot \alpha^{\prime}([x])=\sum_{i=1}^{n-1} b_{i} R^{i+1}$ for some choice of $b_{i}, i=1, \cdots, n-1$, in $B$. Thus, $R \cdot \alpha^{\prime}([x]) t([x])=R \cdot d=t([x]) \sum_{i=1}^{n-1} R^{i+1}$, $t([x])$ as above. If $R \cdot d=0$, then $R=0$. Suppose therefore that $R \neq 0$. Then it follows that $R^{k} \neq 0$ for all $k$. For if $R^{k}=0$, then $R^{k-1} d=0$ and hence $R^{k-1}=0$. Now

$$
\left.\left\|(R d)^{k}\right\|\right|^{1 / k} \leqq K\left\|R^{k}\right\|^{2 / k}
$$

where $K=\left\|t([x]) \cdot \sum_{i=1}^{n-1} b_{i} R^{i-1}\right\| \neq 0$. For each integer $k$, we have that $\left\|(R d)^{k} \mid\right\|^{1 / k} \geqq \mu\left\|R^{k}\right\|^{1 / k}$. For if $R^{k}=\sum_{i=0}^{n-1} r_{i}^{(k)}[x]^{i}, r_{i}^{(k)} \in \operatorname{Rad}(A) \quad($ recall that $\operatorname{Rad}(B)=(\operatorname{Rad}(A))[[x]]$, then

$$
\left\|(R d)^{k}\right\|^{1 / k}=\left(\sum_{i=0}^{n-1}\left\|r_{i}^{(k)} d^{k}\right\|\right)^{1 / k} \geqq\left(\mu^{k} \sum_{i=0}^{n-1}\left\|r_{i}^{(k)}\right\|\right)^{1 / k}=\mu\left\|R^{k}\right\|^{1 / k} .
$$

Combining the above inequalities, we have

$$
\mu\left\|R^{k}\right\|^{1 / k} \leqq K\left\|R^{k}\right\|^{2 / k} .
$$

Since $R^{k} \neq 0$ for all $k$, we have that $\mu \leqq K\left\|R^{k}\right\|^{1 / k}$. But $R \in \operatorname{Rad}(B)$ so that $\lim _{k \rightarrow \infty}\left\|R^{k}\right\|^{1 / k}=0$. Thus a contradiction and so $R$ must have been zero.

Condition (ii) of the above corollary is satisfied when $d$ is not a topological divisor of zero in $A$ but may still be satisfied if $d$ is a topological divisor of zero in $A$.

The case where the discriminant $d$ of $\alpha(x)$ is invertible in $A$ deserves special attention. In this case, if $f \in C\left(\Phi_{B}\right)$ and $\widehat{\alpha}(f)=0$, then there exists a $b \in B$ such that $\alpha(b)=0$ and $\hat{b}=f$ (cf. 2.4 or [1]). Now, if $\phi \in E\left(\Phi_{B}: \Phi_{A}\right)$, then define $f(h, \lambda)=\mu$ where $(h, \mu)=\phi(h, \lambda)$. It is easily shown that $f$ is a continuous function on $\Phi_{B}$. Since $\alpha(f)=0$, there exists a $b \in B$ with the above properties. Since $\phi$ is one-to-one, $\hat{b}$ $(=f)$ separates the points of $\pi^{-1}(h)$ for each $h \in \Phi_{\Delta}$. Hence, it follows from Theorem 6.3 that $g: \sum_{i=0}^{n-1} a_{i}[x]^{i} \rightarrow \sum_{i=0}^{n-1} a_{i} b^{i}$ is an automorphism of $B$. (Note that $g^{*}=\phi$.) If we write $(*)$ for the mapping $g \rightarrow g^{*}$, $g \in G(B: A)$, then we have

Corollary 6.5. If $d$ is invertible in $A$, then (*): $G(B: A) \rightarrow$ $E\left(\Phi_{B}: \Phi_{A}\right)$ is one-to-one and once.

In closing, we remark that if $g \in G(B: A)$, then $g$ is continuous and 
hence $b i$-continuous.

7. Extensions of ring isomorphisms. If $A$ is a Banach algebra with an involution $(*)$, then we ask: when can $(*)$ be extended to an involution on $A[x] /(\alpha(x))$ ? Or more generally, if $\phi: A_{1} \rightarrow A_{2}$ is a ring isomorphism (need not commute with scalars), $A_{1}$ and $A_{2}$ Banach algebras, when can $\phi$ be extended to a ring isomorphism of $A_{1}[x] /\left(\alpha_{1}(x)\right)$ onto $A_{2}[y] /\left(\alpha_{2}(y)\right)$ (degree $\alpha_{1}(x)=$ degree $\left.\alpha_{2}(y)\right)$ ? Simple examples show that $(*)$ and $\phi$ can not always be extended. However, under the added assumption that the discriminants of $\alpha_{1}(x)$ and $\alpha_{2}(y)$ are invertible in $A_{1}$ and $A_{2}$, respectively, then there is a necessary and sufficient condition that $\phi$ exist. The condition is stated in terms of a topological mapping. The case of extending $\left(^{*}\right)$ is less simple. In the proofs of our results on extending $\left({ }^{*}\right)$ and $\phi$, we must consider elements $b \in A[x] /(\alpha(x))$ such that $\hat{b}$ separates the points of the fibers $\pi^{-1}(h), h \in \Phi_{A}$. We will show that if the discriminant of $\alpha(x)$ is invertible, then such elements generate all of $B$ over $A$. Before we prove this, we state a lemma which says that repeated extensions are algebraic in the strict sense of the word. The lemma is more general than needed here but will be used in the next section.

LemMA 7.1. Let $A$ be a commutative ring (with unit) and let $B_{i}=B_{i-1}\left[x_{i}\right] /\left(\alpha_{i}\left(x_{i}\right)\right), B_{0}=A, i=1,2, \cdots, m$, where $\alpha_{i}\left(x_{i}\right)$ is monic over $B_{i-1}$ for each $i$. If $b \in B_{m}$, then there exists a monic polynomial $\alpha(x)$ over $A$ of degree $n=\prod_{i=1}^{m} n_{i}\left(n_{i}=\operatorname{deg} \alpha_{i}\left(x_{i}\right)\right)$ such that $\alpha(b)=0$.

A proof of this lemma is to be found in [15] (page 255).

THEOREM 7.2. Let $A$ be a Banach algebra and let $\alpha(x) \in A[x]$ be a monic polynomial with an invertible discriminant in $A$. Then $b \in B$ has the property that $A[b]=B$ if and only if $\hat{b}$ separates the points of $\pi^{-1}(h)$ for each $h \in \Phi_{A}$.

Proof. Suppose that $A[b]=B$. Then there are elements $a_{i} \in A$ such that $[x]=\Sigma a_{i} b^{i}$. If $\hat{b}(h, \lambda)=\hat{b}\left(h, \lambda^{\prime}\right)$ where $(h, \lambda)$ and $\left(h, \lambda^{\prime}\right)$ are points in $\Phi_{B}$, then $[x]^{\wedge}(h, \lambda)$ must be equal to $[x]^{\wedge}\left(h, \lambda^{\prime}\right)$ so that $\lambda=\lambda^{\prime}$ since $[x]^{\wedge}$ separates points of $\pi^{-1}(h)$. Hence, $\hat{b}$ separates the points of $\pi^{-1}(h)$ for each $h \in \Phi_{A}$.

Suppose now that $\hat{b}$ separates the points of $\pi^{-1}(h)$ for each $h \in \Phi_{\Delta}$. By Lemma 7.1, we know that $b$ satisfies a monic polynomial $\beta(x)$ of degree $n(=\operatorname{deg} \alpha(x))$. Since for each $h \in \Phi_{A}, \hat{b}$ takes on $n$ distinct values on $\pi^{-1}(h)$, the discriminant of $\beta(x)$ must be invertible in $A$. Let $B_{0}$ denote the extension $A[y] /(\beta(y))$. Then $\Phi_{B_{0}}=\left\{(h, \mu) \in \Phi_{A} \times\right.$ $C:(h, \mu) \beta(y)=0\}$, and $\theta:(h, \lambda) \rightarrow(h, \hat{b}(h, \lambda))$ is a continuous one-to-one 
mapping to $\Phi_{B}$ onto $\Phi_{B_{0}}$ and hence a homeomorphism. Therefore, $[x]^{\wedge} \circ \theta^{-1}$ is a function continuous on $\Phi_{B_{0}}$ and $\hat{\alpha}\left([x]^{\wedge} \circ \theta^{-1}\right)=0$. Hence by the Arens-Calderón theorem (see 2.4 or [1]) there is an element $b_{0} \in B_{0}$ such that $\alpha\left(b_{0}\right)=0$ and $\hat{b}_{0}=[x]^{\wedge} \circ \theta^{-1}$. If $\phi$ denotes the homomorphism

$$
\sum_{i=0}^{n-1} a_{i}[y]^{i} \rightarrow \sum_{i=0}^{n-1} a_{i} b^{i}
$$

and if

$$
b_{0}=\sum_{i=0}^{n-1} a_{i}[y]^{i},
$$

then

$$
\begin{aligned}
\phi\left(b_{0}\right)^{\wedge}(h, \lambda) & =\phi\left(\sum_{i=0}^{n-1} a_{i}[y]^{i}\right)^{\wedge}(h, \lambda)=\left(\sum_{i=0}^{n-1} a_{i} b^{i}\right)^{\wedge}(h, \lambda)=\sum_{i=0}^{n-1} \hat{a}_{i}(h)(\hat{b}(h, \lambda))^{i} \\
& \left.=\sum_{i=0}^{n-1} \hat{a}_{i}(h)([y])^{\wedge}(\theta(h, \lambda))\right)^{i}=\hat{b}_{0}(\theta(h, \lambda))=[x]^{\wedge}(h, \lambda)
\end{aligned}
$$

for all $(h, \lambda) \in \Phi_{B}$. Hence, $\phi\left(b_{0}\right)^{\wedge}=[x]^{\wedge}$ and since $\alpha\left(\phi\left(b_{0}\right)\right)=0$, we have that $\phi\left(b_{0}\right)=[x]$ by 2.4. Thus, $\phi$ is onto and $A[b]=B$.

Corollary 7.3. Maintain the hypotheses on $A$ and $\alpha(x)$. If $f \in C\left(\Phi_{B}\right) \beta(y) \in B[y]$ such that

(i) $\hat{\beta}(f)=0$,

(ii) $f$ separates the points of $\pi^{-1}(h)$ for each $h \in \Phi_{A}$, and

(iii) $M_{\beta}((h, \lambda), f(h, \lambda)) \quad\left(M_{\beta}=\right.$ multiplicity function of $\left.\beta(y)\right)$ is locally constant on $\Phi_{B}$, then there exist $b \in B$ such that $A[b]=B$ and. $\hat{b}=f$.

The corollary follows immediately from 2.5 and the theorem.

Corollary 7.4. Maintain the hypotheses on $A$ and $\alpha(x)$. If $\hat{b}$ separates the points of $\pi^{-1}(h)$ for each $h$ and $\beta(y) \in A[y]$ is a monic polynomial (of degree equal to the degree of $\alpha(x)$ ) satisfied by $b$, then $\phi: \Sigma a_{i}[y]^{i} \rightarrow \Sigma a_{i} b^{i}$ is an isomorphism of $A[y] /(\beta(y))$ onto $A[x] /(\alpha(x))$.

Proof. (We use the notation of the theorem.) By the theorem we know that $A\left[b_{0}\right]=A[y] /(\beta(y))$ so that if $\phi\left(\sum_{i=0}^{n-1} a_{i}[y]^{i}\right)=\phi\left(\sum_{i=0}^{n-1} a_{i}^{\prime} b_{0}^{i}\right)=$ 0 , then $\sum_{i=0}^{n-1} \alpha_{i}^{\prime}[x]^{i}=0$. But this means that $a_{i}^{\prime}=0$ for each $i$ and $\phi$. is an isomorphism.

Note that the above $\phi$ is continuous and hence $b i$-continuous.

Before we state and prove the next result, we require the following comments. Let $g: A_{1} \rightarrow A_{2}$ be a ring isomorphism (onto). Define $g^{*}: \Phi_{A_{1}} \rightarrow \Phi_{A_{2}}$ as follows: for $h \in \Phi_{A_{1}}$, let $g^{*}(h)$ be the linear functional 

and onto, so is $g^{*}$ one-to-one and onto. We now prove

Lemma 7.5. Let $A_{1}$ and $A_{2}$ be Banach algebras. If $g: A_{1} \rightarrow A_{2}$ is a ring isomorphism (onto), then $g^{*}: \Phi_{A_{1}} \rightarrow \Phi_{A_{2}}$ is a homeomorphism induces an isomorphism of $A_{1} / \operatorname{Rad}\left(A_{1}\right)$ onto $A_{2} / \operatorname{Rad}\left(A_{2}\right)$. Now, by a theorem of Kaplansky [9], $A_{1}=\sum_{i=1}^{p} \oplus e_{i} A_{1}$ where the $e_{i}$ are mutually orthogonal idempotents in $A_{1}, e_{i} A_{1} \cong C$ for $i=3,4, \cdots, p$, and $g \mid e_{1} A_{1}$ is linear while $g \mid e_{2} A_{1}$ is conjugate linear. Thus, $\Phi_{A_{1}}=U_{i=1}^{p} \Phi_{e_{i} A_{1}}$ and the $\Phi_{e_{i} A_{1}}$ are disjoint open subsets of $\Phi_{A_{1}}$. Since each $\Phi_{e_{i} A_{1}}$ consists of exactly one point if $3 \leqq i \leqq p, g^{*} \mid U_{i=3}^{p} \Phi_{e_{i} A_{1}}$ is continuous. That $g^{*} \mid \Phi_{e_{1} A_{1}}$ is continuous follows from a now classical result (cf. Theorem $24 B$, [11]). To show shat $g^{*} \mid \Phi_{e_{2} A_{1}}$ is continuous, we take $a \in e_{2} A_{1}$ and let $\lambda=h(a), h \in \Phi_{e_{2} A_{1}}$. Then $\left(a-\lambda e_{2}\right) \in h^{-1}(0)$. Since $g \mid e_{2} A_{1}$ is conjugate linear, $g\left(a-\lambda e_{2}\right)=$ $g(a)-\bar{\lambda} g\left(e_{2}\right) \in g^{*}(h)^{-1}(0)$, and hence $g(a)^{\wedge}\left(g^{*}(h)\right)=(\widehat{a}(h))^{--}$. From this it follows immediately that $g^{*} \mid \Phi_{e_{2} A_{1}}$ is a continuous mapping.

THEOREM 7.6. Let $A_{1}$ and $A_{2}$ be Banach algebras, $\alpha_{1}\left(x_{1}\right) \in A_{1}\left[x_{1}\right]$ and $\alpha_{2}\left(x_{2}\right) \in A_{2}\left[x_{2}\right]$ be monic polynomials with invertible discriminants in $A_{1}$ and $A_{2}$, respectively, and $B_{i}=A_{i}\left[x_{i}\right] /\left(\alpha_{i}\left(x_{i}\right)\right), i=1,2$. If $g$ is a ring isomorphism of $A_{1}$ onto $A_{2}$, then there exists an isomorphism $\widetilde{g}$ of $B_{1}$ onto $B_{2}$ which extends $g$ if and only if there exists a homeomorphism $\gamma$ of $\Phi_{B_{1}}$ onto $\Phi_{B_{2}}$ such that $\pi_{2} \circ \gamma=g^{*} \circ \pi_{1}$, where $\pi_{i}$ is the usual mapping of $\Phi_{B_{i}}$ onto $\Phi_{A_{i}}$. If $g_{1}$ and $g_{2}$ are any two such extensions of $g$, then $g_{1} \circ g_{2}^{-1} \in G\left(B_{2}: A_{2}\right)$.

(Note that if $\gamma$ exists, then $\alpha_{1}\left(x_{1}\right)$ and $\alpha_{2}\left(x_{2}\right)$ must have the same degree since for $h \in \Phi_{A_{1}}, \pi_{1}^{-1}(h)$ and $\pi_{2}^{-1}\left(g^{*}(h)\right)$ have the same number of points.)

Proof. If $\widetilde{g}$ extends $g$, then we take $\gamma=\widetilde{g}^{*}$. By the above lemma, $\gamma$ is a homeomorphism. $\gamma$ is onto since $\widetilde{g}$ is onto. Now, if $M$ is a maximal ideal in $B_{1}$, then

$$
g\left(M \cap A_{1}\right)=\widetilde{g}\left(M \cap A_{1}\right)=\widetilde{g}(M) \cap \widetilde{g}\left(A_{1}\right)=\widetilde{g}(M) \cap A_{2} .
$$

But this means that the restriction of $\widetilde{g}^{*}(h, \lambda)$ to $A_{2}$ is $g^{*}(h)$ if $(h, \lambda)^{-1}(0)=M$. Thus, $\pi_{2} \circ \tilde{g}^{*}=g^{*} \circ \pi_{1}$.

Suppose, now, that $\gamma: \Phi_{B_{1}} \rightarrow \Phi_{B_{2}}$ has the prescribed properties. Let $\beta\left(x_{1}\right)=\sum_{i=0}^{n}\left(g^{-1}\left(\alpha_{2, i}\right)\right) x_{1}^{i}=0$, where $\alpha_{2}\left(x_{2}\right)=\sum_{i=0}^{n} \alpha_{2, i} x_{2}^{i}$. We will show that there is a function $f$ in $B_{1}^{\wedge}$ which separates the points of $\pi_{1}^{-1}(h)$ for each $h$ in $\Phi_{A_{1}}$ and $\hat{\beta}(f)=0$. Let $e_{1}, \cdots, e_{p}$ be the mutually orthogonal idempotents discussed in the proof of the above lemma. We define $f$ 
as follows. If $(h, \lambda) \in \pi_{1}^{-1}\left(\Phi_{e_{1} A_{1}}\right)$, let $f(h, \lambda)=\left[x_{2}\right]^{\wedge}(\gamma(h, \lambda))$ and if $(h, \lambda) \in \pi_{1}^{-1}\left(\Phi_{e_{2} A_{1}}\right)$, let $f(h, \lambda)=\left(\left[x_{2}\right]^{\wedge}(\gamma(h, \lambda))\right)^{-}$. For $h \in U_{i=3}^{p} \Phi_{e_{2} A_{1}}$, let $\mu_{1}(h), \cdots, \mu_{n}(h)$ denote the $n$ distinct roots of $\sum_{i=0}^{n}\left(g^{-1}\left(\alpha_{2, i}\right)\right)^{\wedge}(h) x_{1}^{i}=0$ and let $\left(h, \lambda_{i}(h)\right)$ be the $n$ points in $\pi_{1}^{-1}(h)$. For $\left(h, \lambda_{i}(h)\right)$, let $f\left(h, \lambda_{i}(h)\right)=$ $\mu_{i}(h)$. As defined, $f$ is a continuous function on $\Phi_{B_{1}}$ and satisfies $\widehat{\beta}\left(x_{1}\right)=0$. Since $f$ separates the points of $\pi_{1}^{-1}(h)$ for each $h \in \Phi_{A_{1}}$, and since $\beta(x)$ has an invertible discriminant in $A_{1}$, the Arens-Calderon theorem tells us that there exists $b \in B_{1}$ such that $\hat{b}=f$ and $\sum_{i=0}^{n} g^{-1}\left(\alpha_{2, i}\right) b^{i}=0$. It follows from Corollary 7.4 that $A_{1}[b]=B_{1}$ and $B_{1}$ is isomorphic to $B_{0}=A_{1}[y] /\left(\Sigma g^{-1}\left(\alpha_{2, i}\right) y^{i}\right)$. But $B_{0}$ is, of course, isomorphic to $B_{2}=$ $A_{2}\left[x_{2}\right] /\left(\alpha_{2}\left(x_{2}\right)\right)$ so that $B_{1}$ and $B_{2}$ are isomorphic.

Suppose, now, that $g_{1}$ and $g_{2}$ are any two extensions of $g$. Then $g_{1} \circ g_{2}^{-1}$ is clearly an automorphism of $B_{2}$ onto itself. Since $g_{1}=g_{2}$ on $A_{1}, g_{1} \circ g_{2}^{-1}$ leaves $A_{2}$ invariant elementwise, that is, $g_{1} \circ g_{2}^{-1} \in G\left(B_{2}: A_{2}\right)$.

The above theorem has the following interesting consequence if $A$ is the group algebra $L^{1}(G), G=$ integers. Let $\alpha(x) \in A[x]$ be an irreducible monic polynomial with an invertible discriminant. The irreducibility of $\alpha(x)$ together with the fact that the discriminant is invertible imply that $\Phi_{B}$ is connected (cf. Theorem 2.4, [10]). Then the above theorem implies that $A[x] /(\alpha(x))$ and $A[x] /\left(x^{n}-a_{0}\right)$ are isomorphic, where $n=$ degree $\alpha(x)$ and $a_{0} \in A$ is the unique element such that $\widehat{a}_{0}(z)=z$, $z \in\{\mu \in C:|\mu|=1\}=\Phi_{A}$. If $a \in A$, let $\phi(\alpha)=b$ where $\hat{b}(z)=\sum_{i=-\infty}^{\infty} b_{i} z^{n i}$ and $\hat{a}(z)=\sum_{i=-\infty}^{\infty} b_{i} z^{i}$. Then $\tilde{\phi}: \sum_{i=0}^{n=1} a_{i}[x]^{i} \rightarrow \sum_{i=0}^{n-1} \phi\left(a_{i}\right) a_{\jmath}^{i}$ is clearly an isomorphism of $A[x] /\left(x^{n}-a\right)$ onto $A$ so that $A[x] /(\alpha(x))$ is isomorphic to $A=L^{1}(G)$.

Another interesting consequence is that if $\alpha(x) \in A[x]$ is a monic polynomial with an invertible discriminant, then $A[x] /(\alpha(x))$ is isomorphic to $A[x] /(\alpha(x)+R(x))$ where $R(x) \in(\operatorname{Rad} A)[x]$ and $\operatorname{deg} R(x)<\operatorname{deg} \alpha(x)$.

We now turn our attention to the case where $g: A \rightarrow A$ is a periodic automorphism and, in particular, an involution of a certain type. The following example shows that not every such automorphism is extendable. Let $A=C\left(\{z \in C:|z+1|=1\right.$ or $|z-1|=1\}$ and $\alpha(x)=x^{2}-f, f(z)=$ $z+1$ if $|z+1|=1$ and $f(z)=1$ if $|z-1|=1$. For an involution, we take $f^{*}(z)=(f(-z))^{-}$. $g$ has no extension to $B$ since this would imply that there exists a homeomorphism $\gamma$ of $\Phi_{B}$ onto $\Phi_{B}$ such that $\gamma(z, \lambda)=\left(-z,[x]^{\wedge}(\gamma(z, \lambda))\right.$. But it is impossible for such a homeomorphism to exist. Hence, $g$ has no extension.

However, if $g: A \rightarrow A$ is a periodic automorphism which has an extension $\widetilde{g}$ to $B$ (we are assuming that $\alpha(x)$ has an invertible discriminant), then $\widetilde{g}$ is periodic and its period divides $n ! p, p=$ period of $g$. For if $g^{p}=$ identity automorphism, then $\widetilde{g}^{* p}(h, \lambda)=\left(g^{* p}(h),[x]^{\wedge}\left(\widetilde{g}^{* p}(h, \lambda)\right)\right)=$ $\left(h,[x]^{\wedge}\left(\widetilde{g}^{* p}(h, \lambda)\right)\right.$ so that $\widetilde{g}^{* p} \in E\left(\Phi_{B}: \Phi_{A}\right)$. Hence $\left(\widetilde{g}^{* p}\right)^{n !}=$ identity homomorphism. Thus, $\widetilde{g}^{*}$ is periodic. By Corollary $6.4, \widetilde{g}^{p n !}$ is the identity automorphism. Simple examples show that the period of $\widetilde{g}$ 
may be $p \cdot n$ ! We now restrict our attention to the case where $g$ is a symmetric involution, that is, $\left(a^{*}\right)^{\wedge}(h)=(\widehat{a}(h))^{-}$.

THEOREM 7.6. Let $A$ be a Banach algebra and $\alpha(x) \in A[x]$ a monic polynomial with an invertible discriminant in $A$. If $\left(^{*}\right): A \rightarrow A$ is a symmetric involution, then there exists a unique symmetric involution $\left({ }^{\prime}\right): B \rightarrow B$ which extends (*). If $\left({ }^{\prime \prime}\right)$ is any involution extending (*), then $\left({ }^{\prime \prime}\right)=\left({ }^{\prime}\right) \circ g$ for some $g \in G(B: A)$ which is of period two.

Proof. Let $\alpha^{*}(x)=\sum_{i=0}^{n} \alpha_{i}^{*} x^{i}$ where $\alpha(x)=\sum_{i=0}^{n} \alpha_{i} x^{i}$. Then $\hat{\alpha}^{*}(f)=$ 0 where $f(h, \lambda)=\bar{\lambda}$. By the Arens-Calderón theorem, there is an element $b_{0} \in B$ such that $\alpha^{*}\left(b_{0}\right)=0$ and $\hat{b}_{0}=f$. Let $(')$ denote the mapping defined by $\left(\sum_{i=0}^{n-1} a_{i}[x]^{i}\right)^{\prime}=\sum_{i=0}^{n-1} a_{i}^{*} b_{0}^{i}$. Clearly (') is a homomorphism and $\alpha\left(b_{0}^{\prime}\right)=0$. But

$$
\begin{aligned}
\left(b_{0}^{\prime}\right)^{\wedge}(h, \lambda) & =\left(\sum_{i=0}^{n-1} a_{i}^{*} b_{0}^{i}\right) \hat{(}(h, \lambda)=\left(\sum_{i=0}^{n-1}\left(\widehat{a}_{i}(h)\right)^{-}(\bar{\lambda})^{i}\right) \\
& =\left(\sum_{i=0}^{n-1}\left(\hat{a}_{i}(h)\right) \lambda^{i}\right)^{-}=\lambda=[x]^{\wedge}(h, \lambda)
\end{aligned}
$$

where $b_{0}=\sum_{i=0}^{n-1} a_{i}[x]^{i}$, and $(h, \lambda)$ is any point of $\Phi_{B}$. Thus, $\left(b_{0}^{\prime}\right)^{\wedge}=$ $[x]^{\wedge}$, and it follows that $b_{0}^{\prime}=[x]$. Thus, $\left(^{\prime}\right)$ is an involution. That (') is symmetric follows from the fact that $\left(\left(\Sigma a_{i}[x]^{i}\right)^{\prime}\right)^{\wedge}=\Sigma\left(\left(\hat{a}_{i}\right)^{-}\right) f^{i}, f=$ $\left([x]^{\wedge}\right)^{-}$.

If $\left({ }^{\prime \prime}\right)$ is any symmetric involution on $B$ which extends $\left({ }^{*}\right)$, then $\alpha^{*}\left([x]^{\prime \prime}\right)=0$. But $\left([x]^{\prime \prime}\right)^{\wedge}=\hat{b}_{0}$ so that $[x]^{\prime \prime}=b_{0}$. Thus $\left(^{\prime}\right)$ is a unique symmetric involution extending $(*)$.

If $\left({ }^{\prime \prime}\right)$ is any involution (not necessarily symmetric), then $\left({ }^{\prime}\right)^{-1} \circ\left({ }^{\prime \prime}\right)=$ $g$ belongs to $G(B: A)$. To show $g$ is of period two, consider the following. Since the involution defined on $B^{\wedge}$ by conjugation commutes with every involution, $g^{2}(b)^{\wedge}$ is equal to $\hat{b}$ for every $b \in B$; hence, in particular, $g^{2}([x])^{\wedge}=[x]^{\wedge}$. But $\alpha\left(g^{2}([x])\right)=0$ so that $g^{2}([x])=[x]$ and $g$ is of period two.

8. Primitive elements in repeated extensions. As seen in $\S 6$, there is some analogy between the present study and the classical case of field extensions. We carry this analogy one step further by proving a theorem about the existence of primitive elements in repeated extensions. It will follow from our theorem, that if $\alpha(x)$ is a monic polynomial with an invertible discriminant, then there exists an extension of the form $A[x] /(\beta(x))$ over which $\alpha(x)$ factors into linear factors.

THeOREm 8.1. Let $A$ be a Banach algebra. If $B_{0}=A$ and $B_{i}=$ $B_{i-1}\left[x_{i}\right] /\left(\alpha_{i}\left(x_{i}\right)\right), i=1,2, \cdots, m$, where $x_{i}$ is an indeterminate over $B_{i-1}$ and $\alpha_{i}\left(x_{i}\right) \in B_{i-1}\left[x_{i}\right]$ is a monic polynomial with an invertible discrimi- 
nant in $B_{i-1}$, then there exists a monic polynomial $\alpha(x) \in A[x]$ with an invertible discriminant and an element $b \in B_{m}$ such that $\alpha(b)=0$ and $A[b]=B_{m} \cong A[x] /(\alpha(x))$ (algebraically and topologically).

Proof. The proof is by induction. We shall prove the case $m=2$. Consider $\left[x_{1}\right]^{\wedge}(h, \lambda)+c\left[x_{2}\right]^{\wedge}(h, \lambda, \mu)=\lambda+c \mu$, where $c$ is a complex number, and $(h, \lambda, \mu) \in \Phi_{B_{2}}$. We will show that we can choose $c>0$ such that $\lambda+c \mu \neq \lambda^{\prime}+c \mu^{\prime}$ if $(h, \lambda, \mu) \neq\left(h, \lambda^{\prime}, \mu^{\prime}\right)$. If

$$
F(h)=\min \left\{\left|\lambda-\lambda^{\prime}\right|:(h, \lambda),\left(h, \lambda^{\prime}\right) \in \Phi_{B_{1}} \text { and } \lambda \neq \lambda^{\prime}\right\} \text { for each } h \in \Phi_{A},
$$

then $F$ is a continuous function on $\Phi_{A}$ since $\alpha_{1}\left(x_{1}\right)$ has no singular points in $\Phi_{A}$. Since $\Phi_{A}$ is compact and since $F(h)>0$ for each $h \in \Phi_{A}$, there exists $s>0$ such that $F(h)>s$ on $\Phi_{A}$. Choose $c>0$ so that $s>$ $2 \cdot c \cdot\left\|\left[x_{2}\right]^{\wedge}\right\|_{\infty}$. For this choice of $c$, let $b=\left[x_{1}\right]+c\left[x_{2}\right]$. Now, if $(h, \lambda, \mu) \neq$ $\left(h, \lambda, \mu^{\prime}\right)$, then $\hat{b}(h, \lambda, \mu) \neq \hat{b}\left(h, \lambda, \mu^{\prime}\right)$ and if $(h, \lambda) \neq\left(h, \lambda^{\prime}\right)$, then

$$
\begin{aligned}
&\left|\hat{b}(h, \lambda, \mu)-\hat{b}\left(h, \lambda^{\prime}, \mu^{\prime}\right)\right| \geqq\left|\lambda-\lambda^{\prime}\right|-c \cdot\left|\mu-\mu^{\prime}\right| \\
& \geqq s-c \cdot\left|\mu-\mu^{\prime}\right|>s-2 \cdot c \cdot\left\|\left[x_{2}\right]^{\wedge}\right\|_{\infty}>0 .
\end{aligned}
$$

From this it follows that if $\alpha(x)$ is the monic polynomial (constructed in Lemma 7.1) of degree $n=n_{1} n_{2}$ satisfied by $b$, then its discriminant is invertible since corresponding to each $h, \alpha_{h}(x)=0$ has $n_{1} n_{2}$ distinct roots.

Let $B=A[x] /(\alpha(x))$. Then $\Phi_{B}$ is (identifiable with) $\left\{(h, \lambda) \in \Phi_{A} \times C\right.$ : $(h, \lambda) \alpha(x)=0\}$. Hence $\gamma:(h, \lambda, \mu) \rightarrow(h, \hat{b}(h, \lambda, \mu))$ is a homeomorphism of $\Phi_{B_{2}}$ onto $\Phi_{B}$. Thus, $\left[x_{1}\right]^{\wedge} \circ \gamma^{-1}$ is continuous on $\Phi_{B}$ and $\widehat{\alpha}_{1}\left(\left[x_{1}\right]^{\wedge} \circ \gamma^{-1}\right)=$ 0 . By the Arens-Calderón theorem, there exists $b_{1} \in B$ such that $\hat{b}_{1}=$ $\left[x_{1}\right]^{\wedge} \circ \gamma^{-1}$ and $\alpha_{1}\left(b_{1}\right)=0$. Now, if $g: \sum_{i=0}^{n-1} a_{i}[x]^{i} \rightarrow \sum_{i=0}^{n-1} a_{i} b_{1}^{i}$, then $g$ is a homomorphism of $B$ onto $A[b]$. By an argument in the proof of Theorem 7.2, we have that $g\left(b_{1}\right)^{\wedge}=\left[x_{1}\right]^{\wedge}$. But $\alpha_{1}\left(b_{1}\right)=0$ so that $\alpha_{1}\left(g\left(b_{1}\right)\right)=$ 0 from which it follows that $g\left(b_{1}\right)=\left[x_{1}\right]$ since the discriminant of $\alpha_{i}\left(x_{1}\right)$ is invertible. Thus, $A[b]$ contains $\left[x_{1}\right]$ and hence $\left[x_{2}\right] \in A[b]$, i.e., $A[b]=$ $B_{2}$. It remains to show that $g$ is one-to-one and $b i$-continuous. Clearly, $g \mid A\left[b_{1}\right]$ is one-to-one so that there is an element $b_{2} \in B$ which satisfies $\sum_{i=0}^{n_{2}}\left(g \mid A\left[b_{1}\right]\right)^{-1}\left(\alpha_{1}^{(2)}\right) b_{2}^{i}=0$ and $\hat{b}_{2}=\left[x_{2}\right]^{\wedge} \circ \gamma^{-1}$, where $\alpha_{2}(x)=\sum_{i=0}^{n_{2}} \alpha_{i}^{(2)} x^{i}$. As before, $\left.\alpha_{2}\left(g_{2}\right)\right)=0$ and $g\left(b_{2}\right)^{\wedge}=\left[x_{2}\right]^{\wedge}$ so that $g\left(b_{2}\right)=\left[x_{2}\right]$. Hence, $g \mid A\left[b_{1}+c b_{2}\right]$ is a one-to-one mapping. But $\left(b_{1}+c b_{2}\right)^{\wedge}=[x]^{\wedge}$ so that $A\left[b_{1}+c b_{2}\right]=A[x] /(\alpha(x))$. Thus, $g$ is one-to-one. (Note that this means that $b_{1}+c b_{2}=[x]$.) The continuity of $g$ follows as in Theorem 7.2. The $b i$-continuity follows from the closed graph theorem.

COROLlaRY 8.2. If $\alpha(x) \in A[x]$ is a monic polynomial with an invertible discriminant in $A$, then there exists an extension of the form $A[x] /(\beta(x))$ over which $\alpha(x)$ factors into linear factors, where 
$\beta(x)$ is a monic polynomial with an invertible discriminant.

In view of the theorem, the proof of the corollary follows from the fact that if $\alpha(x)=\left(x-b_{1}\right), \cdots,\left(x-b_{t}\right) Q(x)$ over $A[x] /(\alpha(x))$, then $Q(x)$ must have an invertible discriminant over $A[x] /(\alpha(x))$.

9. On the radical of $B$. Let $A$ be a normed algebra and let $K(A)$ denote the intersection of the closed maximal ideals of $A$. If $K(A)=$ (0), we say that $A$ is tractable. In [2] it is shown that if $A$ is tractable and if the discriminant of $\alpha(x)$ is not a zero divisor in $A$, or zero, then $B$ is also tractable. It is further shown that if $A$ is tractable and if $\alpha(x)=x^{n}-a$, then $B$ is tractable if and only if $\alpha$ is not a zero divisor in $A$, or zero. Actually, these results are true for a wider class of algebras, namely, commutative algebras (with unit) over fields of characteristic zero, with "tractable" replaced by "semi-simple."

In this section, we will show that the converse of the above theorem is also valid; indeed, we formulate our theorems and corollaries in the general context of algebras over fields of characteristic zero. To do so requires no extra effort, except that of characterizing the maximal ideals of $B$ in terms of those of $A$. It will follow from the general results presented that when $A$ is tractable, then the radical of $B$ and the intersection of the closed maximal ideals of $B$ coincide, a result that is generally not valid for normed algebras. (An example of a semi-simple normed algebra which is not tractable is given at the end of this section.) Thus, until further notice, we assume that $A$ is a commutative algebra (with unit) over a field $F$ of characteristic zero. Let $M_{A}$ denote the maximal ideal space of $A$. We first identify $M_{B}$ in terms of $M_{A}$. If $m_{0}$ is a maximal ideal in $B$, then $B / m_{0}$ is a field which contains an isomorphic copy of $F$ and hence is also of characteristic zero. Let $\phi$ denote the canonical homomorphism of $B$ onto $B / m_{0}$. Then $\phi(A)$ is a subfield of $B / m_{0}$ since the latter is a simple algebraic extension of $\phi(A)$ (cf. page 259, [15]). Thus we see that $m_{0} \cap A$ is a maximal ideal of $A$. On the other hand, if $m$ is a maximal ideal in $A$, then we can extend $m$ to (at most $n=$ degree of $\alpha(x)$ ) a maximal ideal of $B$. We proceed to show this assertion and at the same time give a description of the extensions.

If $I$ is an ideal in $A$, then let $\beta_{I}(x)$ denote $\Sigma\left(\beta_{i}+I\right) x^{i}$ where $\beta(x)=\Sigma \beta_{i} x^{i}$.

Let $m \in M_{A}$ and $\gamma(x)$ denote a monic polynomial over $A$ such that $\gamma_{m}(x)$ is an irreducible factor of $\alpha_{m}(x)$. Let $(m, \gamma(x))$ denote the set

$$
\left\{\left(\sum_{i=0}^{n-1} a_{i}[x]^{i}\right) \gamma([x])+\sum_{i=0}^{n-1} m_{i}[x]^{i}: a_{i} \in A, m_{i} \in m\right\} \text {. }
$$

It is clear that $(m, \gamma(x))$ is an ideal in $B$. If we define $\theta$ by 


$$
\theta\left(\sum_{i=0}^{n-1} a_{i}[x]^{i}\right)=\sum_{i=0}^{n-1}\left(a_{i}+m\right)\left(x+\left(\gamma_{m}(x)\right)\right)^{i}
$$

then $\theta$ is a homomorphism of $B$ onto $(A / m)[x] /\left(\gamma_{m}(x)\right)$. Clearly $(m, \gamma(x)) \subseteq$ $\theta^{-1}(0)$. Now if $\sum_{i=0}^{n-1}\left(a_{i}+m\right)\left(x+\left(\gamma_{m}(x)\right)\right)^{i}=0$, then

$$
\sum_{i=0}^{n-1}\left(a_{i}+m\right) x^{i}=\gamma_{m}(x) Q_{m}(x),
$$

where $Q(x) \in A[x]$ or equivalently,

$$
\sum_{i=0}^{n-1} a_{i} x^{i}-\gamma(x) Q(x) \in m[x],
$$

Thus, $\sum_{i=0}^{n-1} a_{i}[x]^{i} \in(m, \gamma(x))$. Hence, $\theta^{-1}(0)=(m, \gamma(x))$ and so $(m, \gamma(x))$ is a maximal ideal of $B$.

From the above, it is clear that if $\gamma_{1}(x)-\gamma_{2}(x) \in m[x]$, then $\left(m, \gamma_{1}(x)\right)=\left(m, \gamma_{2}(x)\right)$. We now show the converse. Suppose $\left(m, \gamma_{1}(x)\right)=$ $\left(m, \gamma_{2}(x)\right)$. There exists $p(x) \in A[x]$ and $m(x) \in m[x]$ such that $\gamma_{2}(x)=$ $\gamma_{1}(x) p(x)+m(x)$. Now, $\gamma_{2 m}(x)=\gamma_{1 m}(x) p_{m}(x)$. Since both $\gamma_{1 m}(x)$ and $\gamma_{2 m}(x)$ are irreducible, and monic, $p_{m}(x)=e+m$. The degrees of $\gamma_{1}(x), \gamma_{2}(x)$, $\gamma_{1 m}(x)$ and $\gamma_{2 m}(x)$ are all equal so that $p(x)=e$. Thus, $\gamma_{2}(x)-\gamma_{1}(x) \in m[x]$.

So far we have shown that each maximal ideal of $A$ extends to at least one maximal ideal of $B$. Furthermore, each maximal ideal of $B$ extends a unique maximal ideal of $A$. We shall now show that each maximal ideal $m_{0}$ of $B$ is of the form given above, with $m=m_{0} \cap A$. From earlier comments we know that $B / m_{0}$ is a simple algebraic extension of the field $\phi(A)$, where $\phi: B \rightarrow B / m_{0}$ is the canonical homomorphism. Since $\phi([x])$ is a root of $\alpha_{m}(x)=0, \phi([x])$ must satisfy one of its irreducible factors, say $\beta_{m}(x)$. Hence $B / m_{0}$ must be isomorphic to $\phi(A)[x] /\left(\beta_{m}(x)\right)$. Thus, if $\phi\left(\sum_{i \rightarrow 0}^{n=1} a_{i}[x]^{i}\right)=0$, then $\sum_{i=0}^{n-1}\left(a_{i}+m\right) x^{i}=$ $Q_{m}(x) \beta_{m}(x)$. Thus, $m_{0}=(m, \beta(x))$.

In summary, we have that $M_{B}$ may be viewed as the set of ordered pairs $(m, \beta(x)), m \in M_{A}, \beta(x)$ monic and $\beta_{m}(x)$ an irreducible factor of $\alpha_{m}(x)$. Of course, we identify any two such pairs $(m, \beta(x))$ and $\left(m^{\prime}, \gamma(x)\right)$ if and only if $m=m^{\prime}$ and $\beta(x)-\gamma(x) \in m[x]$. As before, we let $\pi$ denote the (onto) mapping $(m, \beta(x)) \rightarrow m$.

In what follows, let $a(m)$ denote the coset $a+m, a \in A, m \in M_{A}$.

In order to avoid interrupting the proof of the main theorem, we will next state and prove a lemma about the existence of a common factor of $a \alpha(x)$ and $b \alpha^{\prime}(x)$ for suitable elements $a$ and $b$ in $A$. In general, $a$ and $b$ will not be invertible elements (consider the $\alpha(x)$ in Example 5.3). We will need the following result [15]: Let $f(x)$ and $g(x)$ be polynomials over $A$ of respective degrees $m$ and $n$, let $k=$ $\max (m-n+1,0)$ and let $a$ be the leading coefficient of $g(x)$. Then there exist polynomials $Q(x)$ and $R(x)$ over $A$ such that 


$$
a^{k} f(x)=Q(x) g(x)+R(x)
$$

and $R(x)$ is either of degree less than $n$ or is the zero polynomial.

Lemma 9.1. Let $A$ be semi-simple. If the discriminant $d$ of $\alpha(x)$ is a zero divisor in $A$ (say $d c=0, c \neq 0$ ) or if $d=0$, then there are nonzero elements $a$ and $b$ in $A$ and polynomials $\gamma(x), \delta(x)$ and $R(x)$ over $A$ such that

(i ) $\quad \alpha \alpha(x)=\gamma(x) R(x)$

(ii) $b \alpha^{\prime}(x)=\delta(x) R(x)$

(iii) for $m \in M_{A}, a(m)=0$ if and only if $b(m)=0$, and if $c(m)=$ 0 , then $a(m)=0$, and

(iv) if $\beta_{m}(x)\left(m \in M_{A}\right)$ is a factor of $\alpha_{m}(x)$ and $\alpha_{m}^{\prime}(x)$, then $\beta_{m}(x)$ is a factor of $R_{m}(x)$.

Proof. We first prove the lemma for the case $d=0$. Let $R_{-1}(x)$ and $R_{0}(x)$ denote $\alpha(x)$ and $\alpha^{\prime}(x)$, respectively. In view of the above quoted result, we assume that we have found polynomials $Q_{j+1}(x)$, $R_{j+1}(x), 0 \leqq j \leqq i$, over $A$ such that

$$
R_{0 j}^{2 k_{j}} R_{j-1}(x)=R_{0, j-1} R_{j}(x) Q_{j+1}(x)+R_{j+1}(x)
$$

and $R_{0, j} R_{j+1}(x) \neq 0$ for $0 \leqq j \leqq i$, where $R_{0, j}$ denotes the leading coefficient of $R_{0, j-1} R_{j}(x)$ and $k_{i}=\max \left\{\operatorname{deg}\left(R_{j-1}(x)\right)-\left(\operatorname{deg} R_{0, j-1} R_{j}(x)\right)+1,0\right\}$. The polynomial $R_{0, j} R_{j+1}(x)$ is never a non-trivial constant polynomial. This follows from the fact that if $m \in M_{A}$, then $\alpha_{m}(x)$ and $\alpha_{m}^{\prime}(x)$ have at least one irreducible factor in common since $d(m)=0$ (recall that $A / m$ is a field of characteristic zero). For each $m$, let $\beta_{m}(x)$ be one such factor. Thus, it follows that if $R_{0, j}(m) \neq 0$, then $\beta_{m}(x)$ is a factor of $R_{0, j}(m)\left(R_{j+1}\right)_{m}(x)$. Thus, if $R_{0, j} R_{j+1}(x)$ were a constant, say $c$, then $c(m)=0$ for all $m \in M_{\Delta}$. Since $A$ is semi-simple, $c=0$. From this fact and the fact that degree $R_{j+1}(x)<$ degree $R_{0, j} R_{0, j-1}(x)$, we can conclude that there is a first integer, say $i_{0}$, such that $\left(^{*}\right)$ holds with $j=i_{0}$ and $R_{0, i_{0}} R_{i_{0}+1}(x)=0$. Since the coefficients of $R_{i_{0}+1}(x)$ belong to the same maximal ideals that $R_{0, i_{0}}$ belongs to, we have that $R_{i_{0}+1}(x)$ is the zero polynomial. Hence

$$
R_{0 i_{0}}^{2 k k_{0}} R_{i_{0}-1}(x)=R_{0, i} R_{0, i_{0}-1}(x) Q_{i_{0}+1}(x) .
$$

Let $R(x)=R_{0, i_{0}} R_{0, i_{0}-1} R_{i_{0}}(x), a=\prod_{j=0}^{i_{0}} R_{0, j}^{2 \kappa_{j}}$ and $b=\prod_{j=1}^{i_{0}} R_{j_{j}}^{2 k_{j}}$. Then $a$ and $b$ are nonzero and belong to the same maximal ideals to which $R_{0, j}$ belongs. Now, by repeated substitutions, we find polynomials $\gamma(x)$ and $\delta(x)$ over $A$ such that $a \alpha(x)=\gamma(x) R(x)$ and $b \alpha^{\prime}(x)=\delta(x) R(x)$. From the above it is clear that if $\beta_{m}(x)$ is a factor of $\alpha_{m}(x)$ and $\alpha_{m}^{\prime}(x)$, then it is a factor of $R_{m}(x)$.

If $d c=0(d \neq 0, c \neq 0)$, then let $D$ denote the set of maximal ideals 
of $A$ to which $c$ doesn't belong, and $I$ denote the intersection of the maximal ideals in $D$. By the first part of the proof, there are elements $a^{\prime}, b^{\prime} \in A\left(a^{\prime}, b^{\prime} \notin I\right)$ and polynomials $\widetilde{\gamma}(x), \tilde{\delta}(x)$ and $\widetilde{R}(x)$ over $A$ such that for the cosets $a^{\prime}+I$ and $b^{\prime}+I$ and the polynomials $\widetilde{\gamma}_{I}(x), \widetilde{\delta}_{I}(x)$ and $\widetilde{R}_{I}(x)$, the four conditions of the lemma are fulfilled over $A / I$. It then follows that the same four conditions are fulfilled over $A$ if we take $a=c^{2} a^{\prime}, b=c^{2} b^{\prime}, \gamma(x)=c \widetilde{\gamma}(x), \delta(x)=c \tilde{\delta}(x)$ and $R(x)=c \widetilde{R}(x)$. (Note that $a$ and $b$ are not zero since if so we would have that $a^{\prime}$ and $b^{\prime}$ belong to I.) This completes the proof of the lemma.

It is necessary to introduce the following notation at this point. Let $A$ be semi-simple and $\alpha(x)$ a monic polynomial over $A . \quad M_{\alpha}\left(m, \beta_{m}(x)\right)$ is to denote the power to which $\beta_{m}(x)$ appears in the factorization of $\alpha_{m}(x)$ into irreducible factors. Let $d_{k}$ denote the resultant of $\alpha(x)$ and $\alpha^{(k)}(x)$ (= the formal $k$ th derivative of $\alpha(x)$ ), $1 \leqq k \leqq n-1$ (cf. page $96,[14])$ and let $k(\alpha)$ denote the smallest integer $k$, if it exists, such that $d_{k}$ is not a zero divisor in $A$, or zero, and $n$ if all the $d_{k}$ are zero divisors in $A$, or zero. From the definition it follows that if $k>k(\alpha)$, then $d_{k}$ is not a zero divisor in $A$ or zero.

By a nil ideal in $A$ we mean an ideal all of whose elements are nilpotent. If $I$ is an ideal in $A$ for which there exists an integer $k$ such that $a_{1} \cdot a_{2} \cdots \cdots \cdot a_{k}=0$ whenever $a_{i} \in I, i=1,2, \cdots, k$, then we say that $I$ is nilpotent (and write $I^{k}=(0)$ ) and if $k$ is the smallest such integer, then we call $k$ the degree of nilpotency of $I$.

THEOREM 9.2. Suppose that $A$ is semi-simple and that $\alpha(x)$ is a monic polynomial over $A$ for which $k(\alpha) \geqq 2$. Then the radical of $B$ is nontrivial consisting precisely of the nilpotent elements of $B$. Furthermore, Rad $B$ is nilpotent and its degree of nilpotency is $k(\alpha)$.

Proof. It is well known that the radical of an algebra contains all the nilpotent elements of the algebra. We show that $\operatorname{Rad}(B)$ consists of precisely nilpotent elements by showing the last assertion of the theorem, from which it follows that $\operatorname{Rad}(B)$ is nontrivial.

Suppose that $\beta_{1}([x]), \cdots, \beta_{k(\alpha)}([x]) \in \operatorname{Rad}(B)$ and set $\beta(x)=\prod_{i=1}^{k(\alpha)} \beta_{i}(x)$. Then there are polynomials $Q(x)$ and $R(x)$ over $A$ such that $\beta(x)=$ $\alpha(x) Q(x)+R(x)$, with degree $R(x)<$ degree $\alpha(x)$. We will show that $\beta([x])=0$ by showing that $R(x)$ is the zero polynomial. Suppose first that $m \in M_{A}$ has the property that $M_{\alpha}\left(m, \gamma_{m}(x)\right) \leqq k(\alpha)$ for every irreducible factor $\gamma_{m}(x)$ of $\alpha_{m}(x)$. Since $\beta_{i}([x]) \in \operatorname{Rad}(B)$, we know that $\gamma_{m}(x)$ must divide $\left(\beta_{i}\right)_{m}(x)$, and hence $\gamma_{m}(x)^{j}, j=M_{\infty}\left(m, \gamma_{m}(x)\right)$ divides $\beta_{m}(x)$. Furthermore $\gamma_{m}(x)^{j}$ divides $\alpha_{m}(x)$ (by definition of $j$ ) so that $\gamma_{m}(x)^{j}$ also divides $R_{m}(x)$. But $\gamma_{m}(x)$ is an arbitrary irreducible factor so it follows that $\alpha_{m}(x)$ divides $\beta_{m}(x)$ and consequently also divides $R_{m}(x)$. Since degree $R_{m}(x)<$ degree $\alpha_{m}(x), R_{m}(x)$ is the zero polynomial over $A / m$, or equiva- 
lently, the coefficients of $R(x)$ lie in $m$. If there is a $m \in M_{A}$ such that $M_{\alpha}\left(m, \gamma_{m}(x)\right)>k(\alpha)$ for some $\gamma(x)$, then $d_{k(\alpha)} \in m$. Thus, the coefficients of $d_{k(\alpha)} R(x)$ lie in every maximal ideal in $A$ and hence are all zero. But $d_{k(\alpha)}$ is neither a zero divisor in $A$ or zero, so that $R(x)$ is the zero polynomial over $A$. Thus, $\beta(x)=\alpha(x) Q(x)$, or equivalently, $\beta([x])=$ $\prod_{i=1}^{k(\alpha)} \beta_{i}([x])=0$.

To show that $\operatorname{Rad}(B)^{k(\alpha)-1} \neq(0)$ (recall that $k(\alpha)$ is assumed to be greater than one), it suffices to show that there is an element $f \in \operatorname{Rad}(B)$ such that $f^{k(\alpha)-1} \neq 0$. We will show that $f=a \gamma([x])$ is a suitable choice, where $a$ and $\gamma(x)$ are supplied to us by Lemma 9.1. (We may assume that $c$ in the lemma has the property that $c d_{k(\alpha)-1}=0, c \neq 0$.) Let us first note that $a \gamma([x]) \neq 0$. For if not, then $a \gamma(x)=Q(x) \alpha(x)$ for some $Q(x) \in A[x]$. But $a \alpha(x)=\gamma(x) R(x)$ so that $a^{2} \alpha(x)=Q(x) R(x) \alpha(x)$ or $a^{2}=$ $Q(x) R(x)$. If $c(m)=0$, then $a(m)=0$. If $c(m) \neq 0$, then $d(m)=0$ so that $\alpha_{m}(x)$ and $\alpha_{m}^{\prime}(x)$ have a common factor which is also a factor of $R_{m}(x)$ by (iv) of the lemma. Thus, $a(m)=0$ for all $m \in M_{\Delta}$ and hence $a=0$, which is a contradiction. We show next that $a \gamma([x]) \in \operatorname{Rad}(B)$.

Let $m$ be a maximal ideal such that $a(m) \neq 0$ and $\beta_{m}(x)$ an irreducible factor of $\alpha_{m}(x)$. If $\beta_{m}(x)$ is not a factor of $b(m) \alpha_{m}^{\prime}(x)$, then $\beta_{m}(x)$ is not a factor of $R_{m}(x)$ (cf. lemma). Hence $\beta_{m}(x)$ must be a factor of $\gamma_{m}(x)$. If, on the other hand, $\beta_{m}(x)$ is a factor of $b(m) \alpha_{m}^{\prime}(x)$, then $\beta_{m}(x)$ is a factor of $\alpha_{m}^{\prime}(x)(b(m) \neq 0$ since $a(m) \neq 0)$. Thus, from the lemma, we can conclude that $\beta_{m}(x)^{k}, k=M_{\alpha}\left(m, \beta_{m}(x)\right)-1$, is also a factor of $\alpha_{m}^{\prime}(x)$, hence a factor of $R_{m}(x)$ since $\beta_{m}(x)^{k+1}$ is a factor of $\alpha_{m}(x)$. Thus, $\beta_{m}(x)$ must be a simple factor of $\gamma_{m}(x)$. We can now conclude that $a \gamma([x])$ belongs to every maximal ideal of $B$.

We now show that $(a \gamma([x]))^{k(\alpha)-1} \neq 0$ or equivalently, $a \gamma(x)^{k(\alpha)-1} \neq$ $Q(x) \alpha(x)$ for every $Q(x) \in A[x]$. Since $k(\alpha) \geqq 2$, we know that there is at least one irreducible factor $\beta_{m}(x)$ of $\alpha_{m}(x)$ for some $m \in M_{A}$ such $\beta_{m}(x)$ is also a factor of $\alpha_{m}^{\prime}(x)$ and $\beta_{m}(x)^{k(\alpha)}$ is a factor of $\alpha_{m}(x)$ (take any $m \in M_{A}$ such that $\left.d_{k(\alpha)-1} \in m\right)$. From what we showed above, we have that $\beta_{m}(x)$ is a simple factor of $\gamma_{m}(x)$. If $(a \gamma(x))^{k(\alpha)-1}=Q(x) \alpha(x)$ for some $Q(x) \in A[x]$, then $\beta_{m}(x)^{k(\alpha)}$ would be a factor of $\left(a(m) \gamma_{m}(x)\right)^{k(\alpha)-1}$ or else $a(m)=0$. Since $a d_{k(\alpha)-1}=0$ (recall our assumption that $c d_{k(\alpha)-1}=0$ ), we may assume that $a(m) \neq 0$. Hence a contradiction since $\beta_{m}(x)$ is only a simple factor of $\gamma_{m}(x)$. Thus, $(a \gamma([x]))^{k(\alpha)-1} \neq 0$.

Corollary 9.3. If $B$ is semi-simple, then $A$ is semi-simple and the discriminant $d$ of $\alpha(x)$ is not a zero divisor in $A$, or zero.

The proof follows immediately from the theorem. To use the theorem, we need to know that $A$ is semi-simple. But this is true since each maximal ideal of $A$ extends to at least one of $B$. This situation is special. (There are examples of semi-simple algebras with 
non-semi-simple subalgebras.)

COROLlaRY 9.4. Let $A$ be a commutative algebra with non-trivial radical $R=\operatorname{Rad}(A)$. Then $\operatorname{Rad}(B)=\left\{b \in B: b^{k} \in R[[x]]\right\}, k=k\left(\alpha_{R}\right)$. If $R$ is a nil ideal, then so is $\operatorname{Rad}(B)$. If $R$ is nilpotent, say $R^{p}=(0)$, then so is $\operatorname{Rad}(B)$ and $\operatorname{Rad}(B)^{p k}=(0)$.

Proof. Since $\operatorname{Rad}(B) \supset R$, it is clear that $\operatorname{Rad}(B) \supseteqq\left\{b \in B: b^{k} \in R[[x]]\right\}$. Now, consider the homomorphism $\phi$ of $B$ onto $(A / R)[x] /\left(\alpha_{R}(x)\right)$ defined by $\phi\left(\Sigma a_{i}[x]^{i}\right)=\Sigma\left(a_{i}+R\right) x^{i}+\left(\alpha_{R}(x)\right)$. Then $\phi(\operatorname{Rad}(B)) \cong \operatorname{Rad}\left((A / R)[x] /\left(\alpha_{R}(x)\right)\right)$ (cf. page 10, [8]). The kernel of $\phi$ is $R[[x]]$. Thus, if $b \in \operatorname{Rad}(B)$, then $\phi\left(b^{k}\right)=(\phi(b))^{k}=0$ by the theorem. It follows that $b^{k} \in \phi^{-1}(0)$ so that $\operatorname{Rad}(B) \subseteq\left\{b \in B: b^{k} \in R[[x]]\right\}$. Thus equality holds and the first assertion of the corollary is established.

Suppose now that $R$ is a nil ideal. Let $b \in \operatorname{Rad}(B)$. Then by the above, $b^{k\left(\alpha_{R}\right)} \in R[[x]]$. Let $b^{k\left(\alpha_{R}\right)}=\sum_{i=0}^{n-1} b_{i}[x]^{i}, b_{i} \in R$. Since $A$ is commutative, the elements $b_{0}, \cdots, b_{n-1}$ generate a nilpotent ideal in $A$ (cf. page 193, [8]). If $p$ is the degree of nilpotency of this ideal, then $\left(b^{k\left(\alpha_{R}\right)}\right)^{p}=0$. Thus, $\operatorname{Rad}(B)$ is a nil ideal.

The last assertion follows immediately from what we just proved.

If the degree of nilpotency of $\operatorname{Rad}(A)$ is $p$, it may well be the case that the degree of nilpotency of $\operatorname{Rad}(B)$ is less than $p k\left(\alpha_{R}\right)$. For example, take an algebra for which $p=2$ and let $\alpha(x)=x^{3}$. Then $\operatorname{Rad}(B)^{4}=\{0\} . \quad$ (It is easy to modify this example so that $\alpha(x)=0$ has no solution in $A$.) On the other hand, the degree of nilpotency of $\operatorname{Rad}(B)$ may be equal to $k\left(\alpha_{R}\right) p$.

We now turn our attention to the case where $A$ is a commutative normed algebra. For such an algebra, $K(A)$ denotes the intersection of its closed maximal ideals.

THeOREM 9.5. Let $A$ be a tractable normed algebra. Then $K(B)$ coincides with the radical of $B$. Hence if $B$ is tractable, then $A$ is tractable and $d$ is not a divisor of zero in $A$, or zero.

In order to prove the theorem we only have to establish that the elements of $K(B)$ are nilpotent. To do this, we must know which maximal ideals of $B$ are closed. Of course, each closed maximal ideal of $B$ extends a maximal ideal of $A$ so that $\Phi_{B}$ (= space of closed maximal ideals of $B)$ is a subset of $D=\left\{(h, \lambda) \in \Phi_{A} \times C: \alpha_{h}(\lambda)=0\right\}$. Actually, $\Phi_{B}=D$. To see this, observe that

$$
\left|\alpha_{h}(\lambda)\right| \geqq|\lambda|^{n}-\left\|\alpha_{n-1}|||\lambda|^{n-1}-\cdots-\right\| \alpha_{1}|||\lambda|-\left\|\alpha_{0}\right\| \text {. }
$$

If $|\lambda|>1$, then the right hand side is greater than zero so that 
$\left|\alpha_{h}(\lambda)\right|>0$ for all $h \in \Phi_{\Delta}$. Thus, if $(h, \lambda) \in D$, then $|\lambda| \leqq 1$ and hence $(h, \lambda)$ defines a continuous multiplicative linear functional (recall that we are assuming that $\left.\left\|\alpha_{0}\right\|+\left\|\alpha_{1}\right\|+\cdots+\left\|\alpha_{n-1}\right\| \leqq 1\right)$.

Now, using the fact that $\Phi_{B}=D$, we use the method of proof of the first assertion of Theorem 9.2 to establish that $K(B)$ is nilpotent. Hence $K(B) \subseteq \operatorname{Rad}(B)$. On the other hand, $\operatorname{Rad}(B) \subseteq K(B)$ so that $K(B)=\operatorname{Rad}(B)$.

The second assertion now follows from Corollary 9.3.

As we have pointed out earlier, there are normed algebras which are semisimple but not tractable. A simple example illustrating this is as follows: Let $A$ be any normed algebra with no nonzero nil ideals but possessing a nontrivial radical. $A[x]$ is a normed algebra under $\left\|\Sigma a_{i} x^{i}\right\|=\Sigma\left\|a_{i}\right\|$. Clearly, $A[x]$ is not tractable. However $A[x]$ is semi-simple (cf. Theorem 4, page 12, [8]).

\section{BIBLIOGRAPHY}

1. R. Arens and A. P. Calderón, Analytic functions of several Banach algebra elements, Ann. of Math., 6 (1955), 204-216.

2. R. Arens and K. Hoffman, Algebraic extensions of normed algebras, Proc. Amer. Math. Soc., 7 (1956), 203-210.

3. W. G. Badé and P. C. Curtis, Jr., The Wedderburn decomposition of commutative Banach algebras, Amer. J. Math., 82 (1960), 851-866.

4. C. Feldman, The Wedderburn principal theorem in Banach algebras, Proc. Amer. Math. Soc., 2 (1951), 771-777.

5. I. Gelfand, D. Raikov and G. Šilov, Commutative normed rings (in Russian), Uspehi Mat. Nauk. (N. S.) 1, No. 2 (12) (1946), 48-146 ; Amer. Math. Soc. Translations (series 2), 5 (1957), 115-220.

6. G. A. Heuer, Algebraic extensions of Banach algebras, Thesis (1958), University of Minnesota.

7. G. A. Heuer and J. A. Lindberg, Algebraic extensions of continuous function algebras, Proc. Amer. Math. Soc., 14 (1963), 337-342.

8. N. Jacobsen, Structure of Rings, Amer. Math. Soc. Coll. Publ. no. 37, Providence (1956).

9. I. Kaplansky, Ring isomorphisms of Banach algebras, Canad. J. Math., 6 (1954), 374-381.

10. J. A. Lindberg, Factorization of Polynomials over Banach algebras, to appear in Trans. Amer. Math. Soc.

11. L. H. Loomis, An Introduction to Abstract Harmonic Analysis, Van Nostrand, New York (1953).

12. C. E. Rickart, General Theory of Banach Algebras, Van Nostrand, New York (1960).

13. G. Šilov, Decomposition of a commutative normed ring (in Russian), Mat. Sbornick N.S., 32 (74) (1953), 353-364; Amer. Math. Soc. Translations (series 2), 5 (1955), 37-48.

14. B. L. Van Der Waerden, Moderne Algebra, Vol. 1 (3rd Ed.), Springer, Berlin (1950).

15. O. Zariski and P. Samuel, Commutative Algebra, Vol. 1, Van Nostrand, New York (1958). 



\title{
PACIFIC JOURNAL OF MATHEMATICS
}

\author{
EDITORS
}

\author{
Robert Osserman \\ Stanford University \\ Stanford, California \\ M. G. Arsove \\ University of Washington \\ Seattle 5, Washington
}

\author{
J. Dugundji \\ University of Southern California \\ Los Angeles 7, California \\ Lowell J. Paige \\ University of California \\ Los Angeles 24, California
}

\section{ASSOCIATE EDITORS}
E. F. BECKENBACH
B. H. NEUMANN
F. WOLF
K. YOSHIDA

\section{SUPPORTING INSTITUTIONS}

\author{
UNIVERSITY OF BRITISH COLUMBIA \\ CALIFORNIA INSTITUTE OF TECHNOLOGY \\ UNIVERSITY OF CALIFORNIA \\ MONTANA STATE UNIVERSITY \\ UNIVERSITY OF NEVADA \\ NEW MEXICO STATE UNIVERSITY \\ OREGON STATE UNIVERSITY \\ UNIVERSITY OF OREGON \\ OSAKA UNIVERSITY \\ UNIVERSITY OF SOUTHERN CALIFORNIA
}

\author{
STANFORD UNIVERSITY \\ UNIVERSITY OF TOKYO \\ UNIVERSITY OF UTAH \\ WASHINGTON STATE UNIVERSITY \\ UNIVERSITY OF WASHINGTON \\ AMERICAN MATHEMATICAL SOCIETY \\ CALIFORNIA RESEARCH CORPORATION \\ SPACE TECHNOLOGY LABORATORIES \\ NAVAL ORDNANCE TEST STATION
}

Mathematical papers intended for publication in the Pacific Journal of Mathematics should by typewritten (double spaced), and on submission, must be accompanied by a separate author's résumé. Manuscripts may be sent to any one of the four editors. All other communications to the editors should be addressed to the managing editor, L. J. Paige at the University of California, Los Angeles 24, California.

50 reprints per author of each article are furnished free of charge; additional copies may be obtained at cost in multiples of 50 .

The Pacific Journal of Mathematics is published quarterly, in March, June, September, and December. Effective with Volume 13 the price per volume (4 numbers) is $\$ 18.00$; single issues, $\$ 5.00$. Special price for current issues to individual faculty members of supporting institutions and to individual members of the American Mathematical Society: $\$ 8.00$ per volume; single issues $\$ 2.50$. Back numbers are available.

Subscriptions, orders for back numbers, and changes of address should be sent to Pacific Journal of Mathematics, 103 Highland Boulevard, Berkeley 8, California.

Printed at Kokusai Bunken Insatsusha (International Academic Printing Co., Ltd.), No. 6, 2-chome, Fujimi-cho, Chiyoda-ku, Tokyo, Japan.

PUBLISHED BY PACIFIC JOURNAL OF MATHEMATICS, A NON-PROFIT CORPORATION

The Supporting Institutions listed above contribute to the cost of publication of this Journal, but they are not owners or publishers and have no responsibility for its content or policies. 


\section{Pacific Journal of Mathematics}

\section{Vol. 14, No. $2 \quad$ June, 1964}

Tom M. (Mike) Apostol and Herbert S. Zuckerman, On the functional equation $F(m n) F((m, n))=F(m) F(n) f((m, n)) \ldots \ldots \ldots \ldots \ldots \ldots \ldots \ldots \ldots \ldots \ldots$

Reinhold Baer, Irreducible groups of automorphisms of abelian groups . . . . . . . 385

Herbert Stanley Bear, Jr., An abstract potential theory with continuous kernel . . . . 407

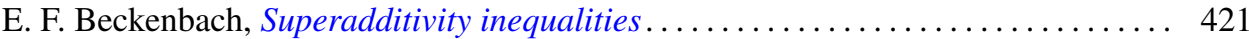

R. H. Bing, The simple connectivity of the sum of two disks . . . . . . . . . . . 439

Herbert Busemann, Length-preserving maps ...................... 457

Heron S. Collins, Characterizations of convolution semigroups of measures . . . . . . 479

Paul F. Conrad, The relationship between the radical of a lattice-ordered group and complete distributivity............................ 493

P. H. Doyle, III, A sufficient condition that an arc in $S^{n}$ be cellular . . . . . . . . . 501

Carl Clifton Faith and Yuzo Utumi, Intrinsic extensions of rings . . . . . . . . . . 505

Watson Bryan Fulks, An approximate Gauss mean value theorem . . . . . . . . . . 513

Arshag Berge Hajian, Strongly recurrent transformations . . . . . . . . . . . . . 517

Morisuke Hasumi and T. P. Srinivasan, Doubly invariant subspaces. II . . . . . . . 525

Lowell A. Hinrichs, Ivan Niven and Charles L. Vanden Eynden, Fields defined by

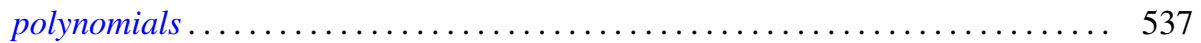

Walter Ball Laffer, I and Henry B. Mann, Decomposition of sets of group

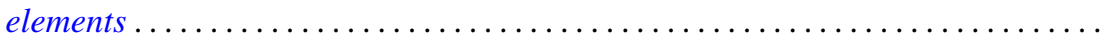

John Albert Lindberg, Jr., Algebraic extensions of commutative Banach

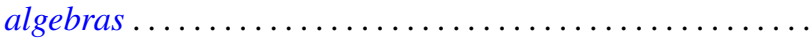

W. Ljunggren, On the Diophantine equation $C x^{2}+D=y^{n} \ldots$

M. Donald MacLaren, Atomic orthocomplemented lattices ....

Moshe Marcus, Transformations of domains in the plane and applications in the

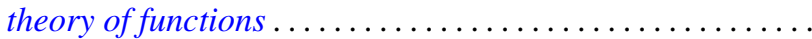

Philip Miles, $B^{*}$ algebra unit ball extremal points . .................. 627

W. F. Newns, On the difference and sum of a basic set of polynomials . . . . . . . 639

Barbara Osofsky, Rings all of whose finitely generated modules are injective ...... 645

Calvin R. Putnam, Toeplitz, matrices and invertibility of Hankel matrices . . . . . . . 651

Shoichiro Sakai, Weakly compact operators on operator algebras . . . . . . . . . 659

James E. Simpson, Nilpotency and spectral operators . . . . . . . . . . . . . 665

Walter Laws Smith, On the elementary renewal theorem for non-identically

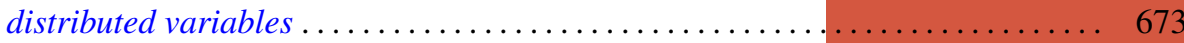

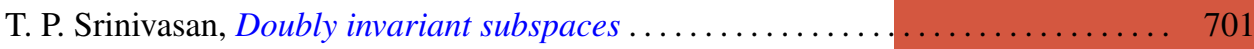

J. Roger Teller, On the extensions of lattice-ordered groups . . . . . . . . . . . . 709

Robert Charles Thompson, Unimodular group matrices with rational integers as

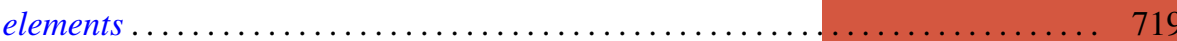

J. L. Walsh and Ambikeshwar Sharma, Least squares and interpolation in roots of unity

Charles Edward Watts, A Jordan-Hölder theorem .................... 731

Kung-Wei Yang, On some finite groups and their cohomology .............. 735

Adil Mohamed Yaqub, On the ring-logic character of certain rings ............ 741

Paul Ruel Young, A note on pseudo-creative sets and cylinders 